Research Article

\title{
Bounds on General Randić Index for F-Sum Graphs
}

\author{
Xu Li $\left(\mathbb{D},{ }^{1}\right.$ Maqsood Ahmad, ${ }^{2,3}$ Muhammad Javaid $\mathbb{D}^{2},{ }^{2}$ Muhammad Saeed, ${ }^{2}$ \\ and Jia-Bao Liu ${ }^{4}$ \\ ${ }^{1}$ Sino-German Institute of Applied Mathematics, Hefei University, Hefei 230601, China \\ ${ }^{2}$ Department of Mathematics, University of Management and Technology, Lahore, Pakistan \\ ${ }^{3}$ Department of Mathematics, COMSATS University Islamabad, Lahore Campus, Lahore 54000, Pakistan \\ ${ }^{4}$ School of Mathematics and Physics, Anhui Jianzhu University, Hefei 230601, China
}

Correspondence should be addressed to Muhammad Javaid; javaidmath@gmail.com

Received 5 June 2020; Accepted 11 August 2020; Published 30 August 2020

Academic Editor: Ji Gao

Copyright $\odot 2020 \mathrm{Xu} \mathrm{Li}$ et al. This is an open access article distributed under the Creative Commons Attribution License, which permits unrestricted use, distribution, and reproduction in any medium, provided the original work is properly cited.

\begin{abstract}
A topological invariant is a numerical parameter associated with molecular graph and plays an imperative role in the study and analysis of quantitative structure activity/property relationships (QSAR/QSPR). The correlation between the entire $\pi$-electron energy and the structure of a molecular graph was explored and understood by the first Zagreb index. Recently, Liu et al. (2019) calculated the first general Zagreb index of the $F$-sum graphs. In the same paper, they also proposed the open problem to compute the general Randić index $R_{\alpha}(\Gamma)=\sum_{u v \in E(\Gamma)}\left[\mathrm{d}_{\Gamma}(u) \times \mathrm{d}_{\Gamma}(v)\right]^{\alpha}$ of the $F$-sum graphs, where $\alpha \in \mathbb{R}$ and $\mathrm{d}_{\Gamma}(u)$ denote the valency of the vertex $u$ in the molecular graph $\Gamma$. Aim of this paper is to compute the lower and upper bounds of the general Randić index for the $F$-sum graphs when $\alpha \in \mathbb{N}$. We present numerous examples to support and check the reliability as well as validity of our bounds. Furthermore, the results acquired are the generalization of the results offered by Deng et al. (2016), who studied the general Randić index for exactly $\alpha=1$.
\end{abstract}

\section{Introduction}

Suppose the ordered pair $(V(\Gamma), E(\Gamma))$ denotes a finite, simple, and connected molecular graph $\Gamma$. The set represented by $V(\Gamma)$ is the vertex set and the set denoted by $E(\Gamma)$, disjoint from $V(\Gamma)$, is the edge set. Vertices of $\Gamma$ correspond to atoms, whereas edges represent bonding between atoms. For any vertex $v \in V(\Gamma)$, the number of vertices adjacent with $v$ is called the degree of vertex $v$ and is denoted by $\mathrm{d}_{\Gamma}(v)$. The smallest and the largest degree of $\Gamma$ are symbolized by $\delta_{\Gamma}$ and $\Delta_{\Gamma}$, respectively. Two primary parameters known as the order (total number of vertices) and the size (total number of edges) of graph $\Gamma$ are denoted by $n$ and $e$. A path $P_{k}$ is a simple graph having order $k$ and size $k-1$ with the property that exactly two vertices have degree 1 , and rest of the vertices have degree 2 . A cycle $C_{m}$ is a simple graph with same order and size $m$ in such a way that each vertex has degree 2.

Graph theory is playing a remarkable role in various domains of science, especially in mathematical chemistry, computer science, and chemical graph theory since the middle of last century. Let $\Omega$ be a collection of simple graphs and $\mathbb{R}$ be a set of real numbers; then, a topological index (TI) is considered to be a function $\Phi: \Omega \longrightarrow \mathbb{R}$ that associates a graph to a real number. It is worth noting that all the TIs are invariant for the isomorphic structures. To probe and study the chemical, structural, and physical properties of the molecular graphs within the subject of chemical graph theory, several TIs are proposed and intensely investigated. These TIs helped to study the chemical reactivities and physical features such as heat of evaporation and formation, boiling, melting and freezing point, volume of air and vapor pressure, surface tension and density, and critical temperature of the chemical compounds that are involved in the molecular graphs. Moreover, medical behaviors of the drugs, nanomaterials, and crystalline materials which are very important for the chemical industries including pharmaceutical are studied using TIs. For further reading regarding development and applications of TIs, the readers are referred to $[1-10]$. 


\section{Preliminaries and Background}

Some convinced and significant degree-based TIs closely related to our work are defined below.

Definition 1. Let $\Gamma$ be a molecular graph; then, the first Zagreb index and second Zagreb index are defined as

$$
\begin{aligned}
& M_{1}(\Gamma)=\sum_{v \in V(\Gamma)}\left[\mathrm{d}_{\Gamma}(v)\right]^{2}=\sum_{u v \in E(\Gamma)}\left[\mathrm{d}_{\Gamma}(u)+\mathrm{d}_{\Gamma}(v)\right], \\
& M_{2}(\Gamma)=\sum_{u v \in E}\left[\mathrm{~d}_{\Gamma}(u) \cdot \mathrm{d}_{\Gamma}(v)\right] .
\end{aligned}
$$

Gutman and Trinajstić [11] defined the first and second Zagreb indices to establish the relationship between the entire $\pi$-electron energy and a structure of a molecular graph.

Definition 2. Let $\Gamma$ be a molecular graph; then, the first general Zagreb index (FGZI) is defined as

$$
M^{\alpha}(\Gamma)=\sum_{v \in V(\Gamma)}\left[\mathrm{d}_{\Gamma}(v)\right]^{\alpha}=\sum_{v w \in E(\Gamma)}\left[\mathrm{d}_{\Gamma}^{\alpha-1}(v)+\mathrm{d}_{\Gamma}^{\alpha-1}(w)\right],
$$

where $\alpha \in \mathbb{R}, \alpha \neq 0$, and $\alpha \neq 1$. Li and Zheng [12] provided the idea of FGZI. It is evident that we achieve $M_{1}(\Gamma)$ (first Zagreb index) and $F(\Gamma)$ (forgotten index) by putting $\alpha=2$, and $\alpha=3$ in FGZI, respectively $[11,13]$.

Definition 3. Let $\Gamma$ be a molecular graph; then, the general Randić index (GRI) is defined as

$$
R_{\alpha}(\Gamma)=\sum_{v w \in E(\Gamma)}\left[\mathrm{d}_{\Gamma}(v) \mathrm{d}_{\Gamma}(w)\right]^{\alpha}, \quad \alpha \in \mathbb{R}, \alpha \neq 0 .
$$

Bollobás and Erdös originated the concept of GRI [14]. It is clear that $\alpha=-1 / 2$ gives the classical Randić connectivity index $R_{-1 / 2}(\Gamma)$ [15] and $\alpha=1$ provides second Zagreb index $M_{2}(\Gamma)[11]$.

Definition 4. Let $\Gamma$ be a molecular graph; then, the general sum-connectivity index (GSCI) is defined as

$$
\chi^{\alpha}(\Gamma)=\sum_{v w \in E(\Gamma)}\left[\mathrm{d}_{\Gamma}(v)+\mathrm{d}_{\Gamma}(w)\right]^{\alpha}, \quad \alpha \in \mathbb{R} .
$$

Zhou and Trinajstić initiated the idea of GSCI [16]. For $\alpha=-1 / 2$, we attain the classical sum-connectivity index (SCI) $\chi^{-1 / 2}(\Gamma)[17]$.

Lučić et al. [18] studied and observed that there is good correlation between indices $R_{-1 / 2}(\Gamma)$ and $\chi^{-1 / 2}(\Gamma)$ themselves besides their correlation with $E_{\pi}(\Gamma)$ ( $\pi$-electron energy) of benzenoid hydrocarbons. For further insight and applications related to all above TIs, the readers are referred to $[19-23]$.

The operations on graphs, in the construction of new graphs, also play an important role in graph theory, where the old graphs are called the factors of the new graph. Cartesian product (binary operation) is an elegant technique to construct a broader network from two base graphs and is inevitable for design and analysis of networks [24]. In [25], Eliasi and Taeri contrived and constructed the $F$-sum graphs $\left(G_{1}+{ }_{F} G_{2}\right)(F \in\{S, R, Q, T\})$ by employing the idea of Cartesian product on graphs $F\left(\Gamma_{1}\right)$ and $\Gamma_{2}$, where $\Gamma_{1}$ and $\Gamma_{2}$ are two simple-connected graphs and $F\left(\Gamma_{1}\right)$ is obtained after applying $F$ on $\Gamma_{1}$ which is elaborated subsequently.

Definition 5. Let $\Gamma$ be a simple, connected, and finite graph; then, the four significant related graphs can be defined as follows $[25,26]$ :

(1) Subdivision graph $S(\Gamma)$ is an expansion of graph $\Gamma$ by introducing an additional vertex on each edge of $\Gamma$.

(2) Triangle parallel graph $R(\Gamma)$ is derived from $S(\Gamma)$ by joining the solid vertices of the original edges of $\Gamma$ that are incident with hollow vertices.

(3) Line superposition graph $Q(\Gamma)$ is obtained from $S(\Gamma)$ by attaching those pairs of new vertices by edges which have common adjacent (solid) vertex.

(4) Total graph $T(\Gamma)$ is constructed from $S(\Gamma)$ by applying $R(\Gamma)$ and $Q(\Gamma)$, simultaneously.

For more details, see Figure 1. For further insight regarding graph operations, see [27].

Definition 6. Let $\Gamma_{1}$ and $\Gamma_{2}$ be two finite, simple, and connected graphs, $F$ be an operation (one of $S, R, Q$, and $T$ ), and $F(\Gamma)$ be a graph (derived from $\Gamma_{1}$ by employing the operation $F$ ) with $V\left(F\left(\Gamma_{1}\right)\right)$ as vertex set and $E\left(F\left(\Gamma_{1}\right)\right)$ as edge set. Then, the $F$-sum graph $\Gamma_{1}{ }_{F} \Gamma_{2}$ is a graph with vertex set $V\left(\Gamma_{1}+{ }_{F} \Gamma_{2}\right)=V\left(F\left(\Gamma_{1}\right)\right) \times\left(V\left(\Gamma_{2}\right)\right)=\left(V\left(\Gamma_{1}\right)\right.$ $\left.\cup E\left(\Gamma_{1}\right)\right) \times\left(V\left(\Gamma_{2}\right)\right)$ such that two vertices $\left(v_{1}, v_{2}\right)$ and $\left(w_{1}, w_{2}\right)$ of $V\left(\Gamma_{1}+{ }_{F} \Gamma_{2}\right)$ are adjacent if and only if $\left[v_{1}=w_{1} \in V(\Gamma)\right.$ and $\left.\left(v_{2}, w_{2}\right) \in E\left(\Gamma_{2}\right)\right]$ or $\left[v_{2}=w_{2} \in V\left(\Gamma_{2}\right)\right.$ and $\left.\left(v_{1}, w_{1}\right) \in E\left(F\left(\Gamma_{1}\right)\right)\right]$.

We observe that the graph $\Gamma_{1}+{ }_{F} \Gamma_{2}$ has $\left|V\left(\Gamma_{2}\right)\right|$ copies of the graph $F\left(\Gamma_{1}\right)$ provided that vertices of these copies are labeled with vertices of $\Gamma_{2}$. In graph $\Gamma_{1}+{ }_{F} \Gamma_{2}$, the vertices of $\Gamma_{1}$ are referred as solid vertices, whereas the vertices $E\left(\Gamma_{1}\right)$ are referred as hollow vertices. Now join only solid vertices having same label in $F\left(\Gamma_{1}\right)$ such that their adjacency in $\Gamma_{2}$ is preserved. For more clarity, see Figure 2.

Following theorems from basic mathematics are of substantial significance in order to obtain core results.

Theorem 1. Binomial and trinomial theorems provide easy and powerful way in expanding expression involving finite higher powers. The algebraic expressions of binomial and trinomial expansions are described beneath, respectively.

$$
\begin{aligned}
\left(x_{1}+x_{2}\right)^{n} & =\sum_{i=0}^{n}\left(\begin{array}{c}
n \\
i
\end{array}\right) x_{1}^{n-i} x_{2}^{i}, \\
\left(x_{1}+x_{2}+x_{3}\right)^{n} & =\sum_{\substack{m, k, l \\
m+k+l=n}} P_{m, k, l} x_{1}^{m} x_{2}^{k} x_{3}^{l},
\end{aligned}
$$

where $P_{m, k, l}=(m+k+l) ! / m ! k ! l !$ 

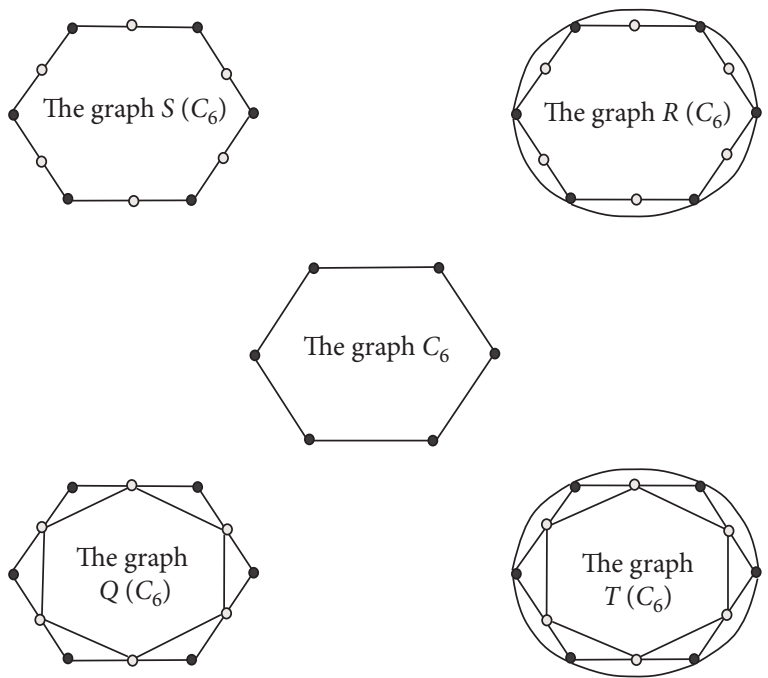

Figure 1: Base graph $C_{6}$ along with its derived graphs.

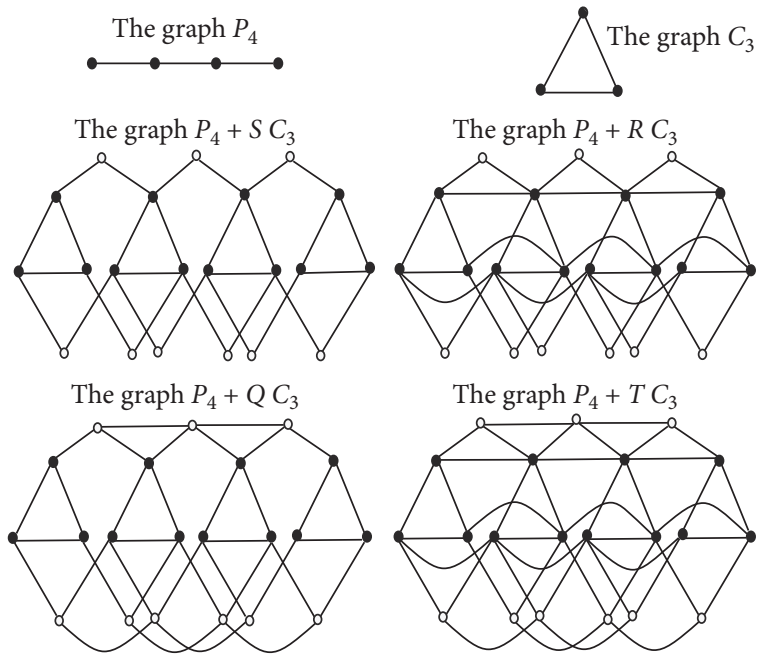

Figure 2: Graphs $P_{5}$ and $P_{6}$ along with their $F$-sum graphs.

Although valency as well as spectral based TIs are current topics of increasing interest for researchers and recently Liu et al [28-30] studied weighted edge corona networks viz a viz spectra of various matrices and valency based indices of Eulerian as well as generalized Sierpinski networks. However, among the valency-based TIs, the Randić index and its variations such as general sumconnectivity, general Randić, harmonic, geometric arithmetic, and atom bond connectivity indices have ample applications in pharmacology and medicinal chemistry [31-33]; for detailed study regarding Randić index, see survey [34]. In [35], Yan et al. computed and analyzed the changes in behavior of Wiener index [36] and enhanced the results to Hosoya polynomial for graph operations presented in Definition 1. In [25], Eliasi and Taeri not only introduced the $F$-sum graphs but also computed the Wiener index of these graphs. Li et al. [37], Shi [38], and Pan et al. [39] provided bounds on Randić index for chemical graphs $\left(d_{v} \leq 4\right)$, bounds on Randić index for triangle-free graph, and sharp bounds on zeroth order general Randić index for unicyclic graphs with fix diameter, respectively. Ali et al. [40], Jamil et al. [41], and Elumalai et al. [42] computed bounds on zeroth order general Randić index for certain type of graphs. Later on, Deng et al. [43], Imran et al. [44], Liu et al. [45], and Ahmad et al. computed the first Zagreb index and second Zagreb index (general Randić index for exactly $\alpha=1$ ) of the $F$-sum graphs, bounds of several indices of the $F$-sum graphs, first generalized Zagreb indices of the $F$-sum graphs, and bounds on general sum-connectivity index for F-sum graphs [46], respectively. Moreover, Liu et al. [45] proposed the open problem to compute the general Randic index for any $\alpha \in R$. In this paper, we solve this open problem, partially, by computing the lower and upper bounds on general Randić index for the $F$-sum graphs for any $\alpha \in \mathcal{N}$.

The rest of the paper is put together as follows. Section 2 covers the materials and methods to determine main results and Section 3 includes some applications of the main results. Section 4 covers the conclusion and further directions of the work.

\section{Results and Discussion}

In this section, the main results regarding general Randic index on the $F$-sum graphs $\Gamma_{1}+{ }_{S} \Gamma_{2}, \Gamma_{1}+{ }_{R} \Gamma_{2}, \Gamma_{1}+{ }_{Q} \Gamma_{2}$ and $\Gamma_{1}+{ }_{T} \Gamma_{2}$, are computed, where $\Gamma_{1}$ and $\Gamma_{2}$ are considered to be finite, simple, and connected graphs. Throughout $n_{1}=V\left(\Gamma_{1}\right), \quad n_{2}=V\left(\Gamma_{2}\right), \quad e_{1}=\left|E\left(\Gamma_{1}\right)\right|, \quad e_{2}=\left|E\left(\Gamma_{2}\right)\right|$, $V\left(S\left(\Gamma_{1}\right)\right)=n_{1}+e_{1}, \quad\left|E\left(S\left(\Gamma_{1}\right)\right)\right|=2 e_{1}, \quad M^{0}\left(\Gamma_{1}\right)=n_{1}$, $M^{1}\left(\Gamma_{1}\right)=2 e_{1}, \quad M^{1}\left(\Gamma_{2}\right)=2 e_{2}, \quad M^{2}\left(\Gamma_{1}\right)=M_{1} \quad\left(\Gamma_{1}\right)$, $M^{2}\left(\Gamma_{2}\right)=M_{1}\left(\Gamma_{2}\right), \quad R_{1}\left(\Gamma_{2}\right)=M_{2}\left(\Gamma_{2}\right), \quad \chi_{0}\left(\Gamma_{2}\right)=e_{2}, \quad$ and $\chi_{1}\left(\Gamma_{1}\right)=M_{1}\left(\Gamma_{1}\right)$.

Theorem 2. Let $\Gamma_{1}$ and $\Gamma_{2}$ be two simple, finite, and connected graphs. For $\alpha \in \mathcal{N}$, the general Randić index of S-sum graph is $L B_{S} \leq R_{\alpha}\left(\Gamma_{1}+{ }_{S} \Gamma_{2}\right) \leq U B_{S}$, where

$$
\begin{aligned}
L B_{S}= & \sum_{\substack{m, k, l \\
m+k+l=n}} P_{m, k, l}\left[M^{2 m+k}\left(\Gamma_{1}\right) \chi_{k}\left(\Gamma_{2}\right)\left(\delta_{\Gamma_{2}}\right)^{2 l}\right] \\
& +\sum_{i=0}^{\alpha}\left(\begin{array}{l}
\alpha \\
i
\end{array}\right)\left[R_{\alpha-i}\left(S\left(\Gamma_{1}\right)\right) M^{i}\left(\Gamma_{2}\right)\left(\delta_{\Gamma_{1}}\right)^{i}\right], \\
U B_{S}= & \sum_{\substack{m, k, l \\
m+k+l=n}} P_{m, k, l}\left[M^{2 m+k}\left(\Gamma_{1}\right) \chi_{k}\left(\Gamma_{2}\right)\left(\Delta_{\Gamma_{2}}\right)^{2 l}\right] \\
& +\sum_{i=0}^{\alpha}\left(\begin{array}{c}
\alpha \\
i
\end{array}\right)\left[R_{\alpha-i}\left(S\left(\Gamma_{1}\right)\right) M^{i}\left(\Gamma_{2}\right)\left(\Delta_{\Gamma_{1}}\right)^{i}\right],
\end{aligned}
$$

and $P_{m, k, l}=(m+k+l) ! / m ! k ! l !$. Equality holds if and only if $\Gamma_{1}$ and $\Gamma_{2}$ are regular graphs with same regularity.

Proof. Suppose that $\mathrm{d}(v, w)=\mathrm{d}_{\left(\Gamma_{1}+\Gamma_{2}\right)}(v, w)$ denotes the degree of vertex $(v, w)$ in the $S$-sum graph $\Gamma_{1}+{ }_{S} \Gamma_{2}$. Then, general Randić index for $S$-sum graph is calculated as 


$$
\begin{aligned}
R_{\alpha}\left(\Gamma_{1}+{ }_{S} \Gamma_{2}\right)= & \sum_{\left(v_{1}, w_{1}\right)}\left[v_{2}, w_{2}\right) \in E\left(\Gamma_{1}+\Gamma_{2}\right) \\
= & \sum_{v \in V\left(\Gamma_{1}\right)} \sum_{w_{1} w_{2} \in E\left(\Gamma_{2}\right)}\left[\mathrm{d}\left(v, v_{1}, w_{1}\right) \mathrm{d}\left(v_{2}, w_{2}\right)\right]^{\alpha} \\
& +\sum_{w \in V\left(\Gamma_{2}\right)} \sum_{v_{1} v_{2} \in E\left(S\left(\Gamma_{1}\right)\right)}\left[\mathrm{d}\left(v_{1}, w\right) \mathrm{d}\left(v_{2}, w\right)\right]^{\alpha} \\
= & \sum 1+\sum 2 .
\end{aligned}
$$

Consider

$$
\begin{aligned}
\sum 1= & \sum_{v \in V\left(\Gamma_{1}\right)} \sum_{w_{1} w_{2} \in E\left(\Gamma_{2}\right)}\left[\left(\mathrm{d}_{\Gamma_{1}}(v)+\mathrm{d}_{\Gamma_{2}}\left(w_{1}\right)\right)\left(\mathrm{d}_{\Gamma_{1}}(v)+\mathrm{d}_{\Gamma_{2}}\left(w_{2}\right)\right)\right]^{\alpha} \\
= & \sum_{v \in V\left(\Gamma_{1}\right)} \sum_{w_{1} w_{2} \in E\left(\Gamma_{2}\right)}\left[\mathrm{d}_{\Gamma_{1}}^{2}(v)+\mathrm{d}_{\Gamma_{1}}(v)\left(\mathrm{d}_{\Gamma_{2}}\left(w_{1}\right)+\mathrm{d}_{\Gamma_{2}}\left(w_{2}\right)\right)\right. \\
& \left.+\mathrm{d}_{\Gamma_{2}}\left(w_{1}\right) \mathrm{d}_{\Gamma_{2}}\left(w_{2}\right)\right]^{\alpha} .
\end{aligned}
$$

Using trinomial theorem, we get

$$
\sum 1=\sum_{v \in V\left(\Gamma_{1}\right)} \sum_{w_{1} w_{2} \in E\left(\Gamma_{2}\right)}\left[\sum_{\substack{m, k, l \\ m, k, l m+k+l=\alpha}} P_{m, k, l} \mathrm{~d}_{\Gamma_{1}}^{2 m}(v) d_{\Gamma_{1}}^{k}(v)\left(\mathrm{d}_{\Gamma_{2}}\left(w_{1}\right)+d_{\Gamma_{2}}\left(w_{2}\right)\right)^{k}\left(\mathrm{~d}_{\Gamma_{2}}\left(w_{1}\right) \mathrm{d}_{\Gamma_{2}}\left(w_{2}\right)\right)^{l}\right],
$$

where $P_{m, k, l}=(m+k+l) ! / m ! k ! l !$.

$$
\sum 1=\sum_{\substack{m, k, l \\ m+k+l=\alpha}} P_{m, k, l}\left[\left(\sum_{w_{1} w_{2} \in E\left(\Gamma_{2}\right)}\left(\mathrm{d}_{\Gamma_{2}}\left(w_{1}\right)+\mathrm{d}_{\Gamma_{2}}\left(w_{2}\right)\right)^{k}\right)\left(\sum_{v \in V\left(\Gamma_{1}\right)} \mathrm{d}_{\Gamma_{1}}^{2 m+k}(v)\right)\left(\mathrm{d}_{\Gamma_{2}}\left(w_{1}\right) \mathrm{d}_{\Gamma_{2}}\left(w_{2}\right)\right)^{l}\right] .
$$

Using Definitions 1 and 3 and the fact Now applying binomial theorem, we get $\delta_{\Gamma_{2}}(w) \leq \mathrm{d}_{\Gamma_{2}}(w) \forall w \in V\left(\Gamma_{2}\right)$, we have

$$
\begin{aligned}
\sum 1 & \geq \sum_{\substack{m, k, l \\
m+k+l=\alpha}} P_{m, k, l}\left[M^{2 m+k}\left(\Gamma_{1}\right) \chi_{k}\left(\Gamma_{2}\right)\left(\delta_{\Gamma_{2}}\right)^{2 l}\right], \\
\sum 2 & =\sum_{w \in V\left(\Gamma_{2}\right)} \sum_{v_{1} v_{2} \in E\left(S\left(\Gamma_{1}\right)\right)}\left[\left(\mathrm{d}\left(v_{1}, w\right) \mathrm{d}\left(v_{2}, w\right)\right]^{\alpha}\right. \\
& =\sum_{w \in V\left(\Gamma_{2}\right)} \sum_{v_{1} v_{2} \in E\left(S\left(\Gamma_{1}\right)\right)}\left[\left(\mathrm{d}_{S\left(\Gamma_{1}\right)}\left(v_{1}\right)+\mathrm{d}_{\Gamma_{2}}(w)\right) \mathrm{d}_{S\left(\Gamma_{1}\right)}\left(v_{2}\right)\right]^{\alpha} \\
& =\sum_{w \in V\left(\Gamma_{2}\right)} \sum_{v_{1} v_{2} \in E\left(S\left(\Gamma_{1}\right)\right)}\left[\mathrm{d}_{S\left(\Gamma_{1}\right)}\left(v_{1}\right) \mathrm{d}_{S\left(\Gamma_{1}\right)}\left(v_{2}\right)+\mathrm{d}_{\Gamma_{2}}(w) \mathrm{d}_{S\left(\Gamma_{1}\right)}\left(v_{2}\right)\right]^{\alpha} .
\end{aligned}
$$

$$
\begin{aligned}
\sum 2 & =\sum_{w \in V\left(\Gamma_{2}\right)} \sum_{S\left(\Gamma_{1}\right)}\left[\sum_{i=0}^{\alpha}\left(\begin{array}{c}
\alpha \\
i
\end{array}\right)\left(\mathrm{d}_{S\left(\Gamma_{1}\right)}\left(v_{1}\right) \mathrm{d}_{S\left(\Gamma_{1}\right)}\left(v_{2}\right)\right)^{\alpha-i}\left(\mathrm{~d}_{\Gamma_{2}}(w) \mathrm{d}_{S\left(\Gamma_{1}\right)}\left(v_{2}\right)\right)^{i}\right] \\
& =\sum_{i=0}^{\alpha}\left(\begin{array}{c}
\alpha \\
i
\end{array}\right)\left[\sum_{v_{1} v_{2} \in E\left(S\left(\Gamma_{1}\right)\right)}\left(\mathrm{d}_{S\left(\Gamma_{1}\right)}\left(v_{1}\right) \mathrm{d}_{S\left(\Gamma_{1}\right)}\left(v_{2}\right)\right)^{\alpha-i}\left(\sum_{w \in V\left(\Gamma_{2}\right)} \mathrm{d}_{\Gamma_{2}}^{i}(w)\right) \mathrm{d}_{S\left(\Gamma_{1}\right)}^{i}\left(v_{2}\right)\right] . \\
& \sum 2 \geq \sum_{i=0}^{\alpha}\left(\begin{array}{c}
\alpha \\
i
\end{array}\right)\left[R_{\alpha-i}\left(S\left(\Gamma_{1}\right)\right) M^{i}\left(\Gamma_{2}\right)\left(\delta_{\Gamma_{1}}\right)^{i}\right] .
\end{aligned}
$$

Using Definitions 5 and 6 and property of smallest degree of graph $\Gamma_{1}$, we have 
Substituting the (12) and (14) in (8), we have

$$
\begin{aligned}
R_{\alpha}\left(\Gamma_{1}+{ }_{S} \Gamma_{2}\right) \geq & \sum_{\substack{m, k, l \\
m+k+l=\alpha}} P_{m, k, l}\left[M^{2 m+k}\left(\Gamma_{1}\right) \chi_{k}\left(\Gamma_{2}\right)\left(\delta_{\Gamma_{2}}\right)^{2 l}\right] \\
& +\sum_{i=0}^{\alpha}\left(\begin{array}{c}
\alpha \\
i
\end{array}\right)\left[R_{\alpha-i}\left(S\left(\Gamma_{1}\right)\right) M^{i}\left(\Gamma_{2}\right)\left(\delta_{\Gamma_{1}}\right)^{i}\right]=L B_{S} .
\end{aligned}
$$

Similarly,

$$
\begin{aligned}
R_{\alpha}\left(\Gamma_{1}+{ }_{S} \Gamma_{2}\right) \leq & \sum_{\substack{m, k, l \\
m+k+l=\alpha}} P_{m, k, l}\left[M^{2 m+k}\left(\Gamma_{1}\right) \chi_{k}\left(\Gamma_{2}\right)\left(\Delta_{\Gamma_{2}}\right)^{2 l}\right] \\
& +\sum_{i=0}^{\alpha}\left(\begin{array}{c}
\alpha \\
i
\end{array}\right)\left[R_{\alpha-i}\left(S\left(\Gamma_{1}\right)\right) M^{i}\left(\Gamma_{2}\right)\left(\Delta_{\Gamma_{1}}\right)^{i}\right]=U B_{S} .
\end{aligned}
$$

Equality holds if and only if $\Gamma_{1}$ and $\Gamma_{2}$ are regular graphs with same regularity. This completes the proof.

Example 1. Let $\Gamma_{1}=P_{4}, \Gamma_{2}=C_{3}, \alpha=2$, and $F=S$. Then, $n_{1}=M^{0}\left(P_{4}\right)=4, \quad n_{2}=M^{0}\left(C_{3}\right)=3, \quad e_{1}=\chi_{0}\left(P_{4}\right)=3$, $e_{2}=\chi_{0}\left(C_{3}\right)=3, \delta_{P_{4}}=1, \Delta_{P_{4}}=2, \delta_{C_{3}}=2$, and $\Delta_{C_{3}}=2$. Moreover, Table 1 contains values of some indices related to certain graphs and is crucial to figure out examples throughout.

Now, we compute lower and upper bound of GRI using formulas derived in Theorem 2 .

$$
\begin{aligned}
L B_{S}\left(P_{4}+{ }_{S} C_{3}\right)= & \sum_{m+k+l=2} P_{m, k, l}\left[M^{2 m+k}\left(P_{4}\right) \chi_{k}\left(C_{3}\right)\left(\delta_{\Gamma_{2}}\right)^{2 l}\right]+\sum_{i=0}^{2}\left(\begin{array}{l}
2 \\
i
\end{array}\right)\left[R_{2-i}\left(S\left(P_{4}\right)\right) M^{i}\left(C_{3}\right)\left(\delta\left(P_{4}\right)\right)^{i}\right] \\
= & P_{1,1,0}\left(M\left(P_{4}\right) \chi\left(C_{3}\right)\right)+P_{1,0,1}\left(M^{2}\left(P_{4}\right) \chi_{0}\left(C_{3}\right)(4)\right)+P_{0,1,1}\left(M\left(P_{4}\right) \chi\left(C_{3}\right)(4)\right) \\
& +P_{2,0,0}\left(M^{4}\left(P_{4}\right) \chi_{0}\left(C_{3}\right)\right)+P_{0,2,0}\left(M^{2}\left(P_{4}\right) \chi_{2}\left(C_{3}\right)\right)+P_{0,0,2}\left(M^{0}\left(P_{4}\right) \chi_{0}\left(C_{3}\right)(16)\right)+\left(R_{2}\left(S\left(P_{4}\right)\right) M^{0}\left(C_{3}\right)\right) \\
& +2\left(R\left(S\left(P_{4}\right)\right) M\left(C_{3}\right)\right)+\left(R_{0}\left(S\left(P_{4}\right)\right) M^{2}\left(C_{3}\right)\right) \\
= & 2(18(12)+10(3)(4)+6(12)(4))+34(3)+10(48)+4(3)(16)+72(3)+2(20)(6)+6(12)=2550 .
\end{aligned}
$$

Likewise, $U B_{S}\left(P_{4}+{ }_{S} C_{3}\right)=3006$.

To calculate exact value for $R_{2}\left(P_{4}{ }_{S} C_{3}\right)$, we require edge partition of graph $P_{4}{ }_{S} C_{3}$, which is presented in Table 2 .

Now, we calculate exact value of GRI of $P_{4}{ }_{5} C_{3}$ for $\alpha=2 . R_{2}\left(P_{4}+{ }_{S} C_{3}\right)=\sum_{v w \in E\left(P_{4}+{ }_{S} C_{3}\right)}\left(\mathrm{d}_{P_{4}}(v) \mathrm{d}_{C_{3}}(w)\right)^{2}=3006$.

Evidently,

$L B_{S}=2550 \leq R_{2}\left(P_{4}+{ }_{S} C_{3}\right)=3006 \leq U B_{S}=3006$.
Additionally, we computed actual values along with corresponding bounds of GRI for various cases, and some are presented in Table 3.

Theorem 3. Let $\Gamma_{1}$ and $\Gamma_{2}$ be two simple, finite, and connected graphs. For $\alpha \in \mathcal{N}$, the general Randic index of $R$-sum graph is $L B_{R} \leq R_{\alpha}\left(\Gamma_{1}+{ }_{R} \Gamma_{2}\right) \leq U B_{R}$, where

$$
\begin{aligned}
L B_{R} & =\sum_{i=0}^{\alpha}\left(\begin{array}{c}
\alpha \\
i
\end{array}\right)\left[2^{(\alpha+i+1)} M^{i}\left(\Gamma_{1}\right) M^{(\alpha-i)}\left(\Gamma_{2}\right)\right]+\sum_{\substack{m, k, l \\
m+k+l=\alpha}} P_{m, k, l} 2^{2 m+k}\left[M^{2 m+k}\left(\Gamma_{1}\right) \chi_{k}\left(\Gamma_{2}\right)\left(\delta_{\Gamma_{2}}\right)^{2 l}+R_{m}\left(\Gamma_{1}\right) M^{k+2 l}\left(\Gamma_{2}\right)\left(2 \delta_{\Gamma_{1}}\right)^{k}\right] \\
U B_{R} & =\sum_{i=0}^{\alpha}\left(\begin{array}{c}
\alpha \\
i
\end{array}\right)\left[2^{(\alpha+i+1)} M^{i}\left(\Gamma_{1}\right) M^{(\alpha-i)}\left(\Gamma_{2}\right)\right]+\sum_{\substack{m, k, l \\
m+k+l=\alpha}} P_{m, k, l} 2^{2 m+k}\left[M^{2 m+k}\left(\Gamma_{1}\right) \chi_{k}\left(\Gamma_{2}\right)\left(\Delta_{\Gamma_{2}}\right)^{2 l}+R_{m}\left(\Gamma_{1}\right) M^{k+2 l}\left(\Gamma_{2}\right)\left(2 \Delta_{\Gamma_{1}}\right)^{k}\right]
\end{aligned}
$$


TABLE 1: Some particular indices of certain graphs.

\begin{tabular}{lcccccccccccc}
\hline Indices/graphs & $M$ & $M^{2}$ & $M^{3}$ & $M^{4}$ & $M^{5}$ & $M^{6}$ & $\chi$ & $\chi_{2}$ & $\chi_{3}$ & $R$ & $R_{2}$ & $R_{3}$ \\
\hline$P_{4}$ & 6 & 10 & 18 & 34 & 66 & 130 & 10 & 34 & 118 & 8 & 24 \\
$P_{5}$ & 8 & 14 & 26 & 50 & 98 & 194 & 14 & 50 & 182 & 12 & 40 & 144 \\
$C_{3}$ & 6 & 12 & 24 & 48 & 96 & 192 & 12 & 48 & 192 & 12 & 48 & 192 \\
$S\left(P_{4}\right)$ & & & & & & & 22 & 82 & 310 & 20 & 72 & 272 \\
$S\left(P_{5}\right)$ & & & & & & & & & & 28 & 104 & 400 \\
\hline
\end{tabular}

TABLE 2: Edge partition based upon degree of end vertices of graph $P_{4}+{ }_{S} C_{3}$.

\begin{tabular}{lcccc}
\hline$\left(d_{v}, d_{w}\right)$ & $(2,3)$ & $(3,3)$ & $(2,4)$ & $(4,4)$ \\
\hline$|E|$ & 6 & 6 & 12 & 6 \\
\hline
\end{tabular}

TABLE 3: Few more cases of lower and upper bounds regarding $R_{\alpha}\left(\Gamma_{1}+{ }_{S} \Gamma_{2}\right)$.

\begin{tabular}{lllr}
\hline$\Gamma_{1}$ & $\Gamma_{2}$ & $\alpha$ & $L B_{S} \leq R_{\alpha}\left(\Gamma_{1}+{ }_{S} \Gamma_{2}\right) \leq U B_{S}$ \\
\hline$P_{4}$ & $C_{3}$ & 3 & $31926 \leq 36390 \leq 36390$ \\
$P_{5}$ & $P_{4}$ & 2 & $3274 \leq 3346 \leq 3850$ \\
$P_{5}$ & $P_{4}$ & 3 & $19941 \leq 34834 \leq 40764$ \\
\hline
\end{tabular}

and $P_{m, k, l}=(m+k+l) ! / m ! k ! l !$. Equality holds if and only if $\Gamma_{1}$ and $\Gamma_{2}$ are regular graphs with same regularity.
Proof. Suppose that $\mathrm{d}(v, w)=\mathrm{d}_{\left(\Gamma_{1}+_{R} \Gamma_{2}\right)}(v, w)$ denotes the degree of vertex $(v, w)$ in the $R$-sum graph $\Gamma_{1}+{ }_{R} \Gamma_{2}$. Then, general Randić index for $R$-sum graph is calculated as

$$
\begin{aligned}
& R_{\alpha}\left(\Gamma_{1}+{ }_{R} \Gamma_{2}\right)=\sum_{\substack{\left(v_{1}, w_{1}\right)\left(v_{2}, w_{2}\right) \\
\in E\left(\Gamma_{1}+{ }_{R} \Gamma_{2}\right)}}\left[\mathrm{d}\left(v_{1}, w_{1}\right) \mathrm{d}\left(v_{2}, w_{2}\right)\right]^{\alpha}=\sum_{v \in V\left(\Gamma_{1}\right) w_{1} w_{2} \in E\left(\Gamma_{2}\right)}\left[\mathrm{d}\left(v, w_{1}\right) \mathrm{d}\left(v, w_{2}\right)\right]^{\alpha} \\
& +\sum_{w \in V\left(\Gamma_{2}\right)} \sum_{v_{1} v_{2} \in E\left(R\left(\Gamma_{1}\right)\right)}\left[\mathrm{d}\left(v_{1}, w\right) \mathrm{d}\left(v_{2}, w\right)\right]^{\alpha} \\
& =\sum_{w_{1} w_{2} \in E\left(\Gamma_{2}\right)}\left[\mathrm{d}\left(v, w_{1}\right) \mathrm{d}\left(v, w_{2}\right)\right]^{\alpha}+\sum_{w \in V\left(\Gamma_{2}\right)} \sum_{\substack{v_{1} v_{2} \in E\left(R\left(\Gamma_{1}\right)\right) \\
v_{1}, v_{2} \in V\left(\Gamma_{1}\right)}}\left[\mathrm{d}\left(v_{1}, w\right) \mathrm{d}\left(v_{2}, w\right)\right]^{\alpha}+\sum_{\substack { w \in V\left(\Gamma_{2}\right) \\
\begin{subarray}{c}{v_{1} v_{2} \in E\left(R\left(\Gamma_{1}\right)\right) \\
v_{1} \in V\left(\Gamma_{1}\right) \\
v_{2} \in V\left(R\left(\Gamma_{1}\right)\right)-V\left(\Gamma_{1}\right){ w \in V ( \Gamma _ { 2 } ) \\
\begin{subarray} { c } { v _ { 1 } v _ { 2 } \in E ( R ( \Gamma _ { 1 } ) ) \\
v _ { 1 } \in V ( \Gamma _ { 1 } ) \\
v _ { 2 } \in V ( R ( \Gamma _ { 1 } ) ) - V ( \Gamma _ { 1 } ) } }\end{subarray}} \\
& \cdot\left[\mathrm{d}\left(v_{1}, w\right) \mathrm{d}\left(v_{2}, w\right)\right]^{\alpha}=\sum 3+\sum 4+\sum 5 \\
& \sum 3=\sum_{v \in V\left(\Gamma_{1}\right)} \sum_{w_{1} w_{2} \in E\left(\Gamma_{2}\right)}\left[\left(\mathrm{d}_{R\left(\Gamma_{1}\right)}(v)+\mathrm{d}_{\Gamma_{2}}\left(w_{1}\right)\right)\left(\mathrm{d}_{R\left(\Gamma_{1}\right)}(v)+\mathrm{d}_{\Gamma_{2}}\left(w_{2}\right)\right)\right]^{\alpha}=\sum_{v \in V\left(\Gamma_{1}\right)} \sum_{w_{1} w_{2} \in E\left(\Gamma_{2}\right)} \\
& \cdot\left[\left(2 \mathrm{~d}_{\Gamma_{1}}(v)+\mathrm{d}_{\Gamma_{2}}\left(w_{1}\right)\right)\left(2 \mathrm{~d}_{\Gamma_{1}}(v)+\mathrm{d}_{\Gamma_{2}}\left(w_{1}\right)\right)\right]^{\alpha} \\
& =\sum_{v \in V\left(\Gamma_{1}\right)} \sum_{w_{1} w_{2} \in E\left(\Gamma_{2}\right)}\left[4 \mathrm{~d}_{G}^{2}(v)+2 \mathrm{~d}_{\Gamma_{1}}(v)\left(\mathrm{d}_{\Gamma_{2}}\left(w_{1}\right)+\mathrm{d}_{\Gamma_{2}}\left(w_{2}\right)\right)+\mathrm{d}_{\Gamma_{2}}\left(w_{1}\right) \mathrm{d}_{\Gamma_{2}}\left(w_{2}\right)^{\alpha}\right] .
\end{aligned}
$$

Applying trinomial theorem, we get 


$$
\sum 3=\sum_{v \in V\left(\Gamma_{1}\right)} \sum_{w_{1} w_{2} \in E\left(\Gamma_{2}\right)}\left[\sum_{\substack{m, k, l \\ m+k+l=\alpha}} P_{m, k, l} 4^{m} \mathrm{~d}_{\Gamma_{1}}^{2 m}(v) 2^{k} \mathrm{~d}_{\Gamma_{1}}^{k}(v)\left(\mathrm{d}_{\Gamma_{2}}\left(w_{1}\right)+\mathrm{d}_{\Gamma_{2}}\left(w_{2}\right)\right)^{k}\left(\mathrm{~d}_{\Gamma_{2}}\left(w_{1}\right) \mathrm{d}_{\Gamma_{2}}\left(w_{2}\right)\right)^{l}\right],
$$

where $P_{m, k, l}=(m+k+l) ! / m ! k ! l !$. Now applying summations on the convenient expressions, then replacing with corresponding formulas, and using smallest degree of graph $\Gamma_{2}$, we get

$$
\begin{aligned}
\sum 3 & =\sum_{\substack{m, k, l \\
m+k+l=\alpha}} P_{m, k, l}\left[2^{2 m+k}\left(\sum_{v \in V\left(\Gamma_{1}\right)} \mathrm{d}_{\Gamma_{1}}^{2 m+k}(v)\right)\left(\sum_{w_{1} w_{2} \in E\left(\Gamma_{2}\right)}\left(\mathrm{d}_{\Gamma_{2}}\left(w_{1}\right)+\mathrm{d}_{\Gamma_{2}}\left(w_{2}\right)\right)^{k}\left(\mathrm{~d}_{\Gamma_{2}}\left(w_{1}\right) \mathrm{d}_{\Gamma_{2}}\left(w_{2}\right)\right)^{l}\right]\right. \\
& \geq \sum_{\substack{m, k, l \\
m+k+l=\alpha}} P_{m, k, l}\left[2^{2 m+k} M^{2 m+k}\left(\Gamma_{1}\right) \chi_{k}\left(\Gamma_{2}\right)\left(\delta_{\Gamma_{2}}\right)^{2 l}\right] .
\end{aligned}
$$

Next sum involves those edges from $R\left(\Gamma_{1}\right)$ whose end vertices are in $V\left(\Gamma_{1}\right)$.

$$
\begin{aligned}
\sum 4 & =\sum_{w \in V\left(\Gamma_{2}\right)} \sum_{\substack{v_{1} v_{2} \in E\left(R\left(\Gamma_{1}\right)\right) \\
v_{1}, v_{2} \in\left(\Gamma_{1}\right)}}\left[\mathrm{d}\left(v_{1}, w\right) \mathrm{d}\left(v_{2}, w\right)\right]^{\alpha} \\
& =\sum_{w \in V\left(\Gamma_{2}\right)} \sum_{\substack{v_{1} v_{2} \in E\left(R\left(\Gamma_{1}\right)\right) \\
v_{1}, v_{2} \in V\left(\Gamma_{1}\right)}}\left[\left(\mathrm{d}_{R\left(\Gamma_{1}\right)}\left(v_{1}\right)+\mathrm{d}_{\Gamma_{2}}(w)\right)\left(\mathrm{d}_{R\left(\Gamma_{1}\right)}\left(v_{2}\right)+\mathrm{d}_{\Gamma_{2}}(w)\right)\right]^{\alpha} \\
& =\sum_{w \in V\left(\Gamma_{2}\right)} \sum_{\substack{v_{1} v_{2} \in E\left(R\left(\Gamma_{1}\right)\right) \\
v_{1}, v_{2} \in V\left(\Gamma_{1}\right)}}\left[\left(2 \mathrm{~d}_{\Gamma_{1}}\left(v_{1}\right)+\mathrm{d}_{\Gamma_{2}}(w)\right)\left(2 \mathrm{~d}_{\Gamma_{1}}\left(v_{2}\right)+\mathrm{d}_{\Gamma_{2}}(w)\right)\right]^{\alpha} \\
& =\sum_{w \in V\left(\Gamma_{2}\right)} \sum_{\substack{v_{1} v_{1} \in E\left(R\left(\Gamma_{1}\right)\right) \\
v_{1}, v_{2} \in V\left(\Gamma_{1}\right)}}\left[4 \mathrm{~d}_{\Gamma_{1}}\left(v_{1}\right) \mathrm{d}_{\Gamma_{1}}\left(v_{2}\right)+2 \mathrm{~d}_{\Gamma_{2}}(w)\left(\mathrm{d}_{\Gamma_{1}}\left(v_{1}\right)+\mathrm{d}_{\Gamma_{1}}\left(v_{2}\right)\right)+\mathrm{d}_{\Gamma_{2}}^{2}(w)\right]^{\alpha} .
\end{aligned}
$$

Now applying trinomial theorem, we have

$$
\begin{aligned}
\sum 4 & =\sum_{w \in V\left(\Gamma_{2}\right)} \sum_{\substack{v_{1} v_{2} \in E\left(R\left(\Gamma_{1}\right)\right) \\
v_{1}, v_{2} \in V\left(\Gamma_{1}\right)}} \sum_{\substack{m, k, l \\
m+k+l=\alpha}} P_{m, k, l}\left(\left(4 \mathrm{~d}_{\Gamma_{1}}\left(v_{1}\right) \mathrm{d}_{\Gamma_{1}}\left(v_{2}\right)\right)^{m} 2^{k} \mathrm{~d}_{\Gamma_{2}}^{k}(w)\left(\mathrm{d}_{\Gamma_{1}}\left(\Gamma_{1}\right)+\mathrm{d}_{\Gamma_{1}}\left(v_{2}\right)\right)^{k}+\mathrm{d}_{\Gamma_{2}}^{2 l}(w)\right) \\
& =\sum_{\substack{m, k, l \\
m+k+l=\alpha}} P_{m, k, l}\left[\sum_{w \in V\left(\Gamma_{2}\right)} \sum_{v_{1} v_{2} \in E\left(\Gamma_{1}\right)}\left(\left(4 \mathrm{~d}_{\Gamma_{1}}\left(v_{1}\right) \mathrm{d}_{\Gamma_{1}}\left(v_{2}\right)\right)^{m} 2^{k} \mathrm{~d}_{\Gamma_{2}}^{k}(w)\left(\mathrm{d}_{\Gamma_{1}}\left(v_{1}\right)+\mathrm{d}_{\Gamma_{1}}\left(v_{2}\right)\right)^{k} \mathrm{~d}_{\Gamma_{2}}^{2 l}(w)\right)\right], \\
\sum 4 & =\sum_{\substack{m, k, l \\
m+k+l=\alpha}} P_{m, k, l}\left[2^{2 m+k}\left(\sum_{\substack{m, k, l \\
v_{1} v_{2} \in E\left(\Gamma_{1}\right)}}\left(\mathrm{d}_{\Gamma_{1}}\left(v_{1}\right) \mathrm{d}_{\Gamma_{1}}\left(v_{2}\right)\right)^{m}\right)\left(\sum_{w \in V\left(\Gamma_{2}\right)} \mathrm{d}_{\Gamma_{2}}^{k+2 l}(w)\right)\left(\mathrm{d}_{\Gamma_{1}}\left(v_{1}\right)+\mathrm{d}_{\Gamma_{1}}\left(v_{2}\right)\right)^{k}\right] \\
& \geq \sum_{\substack{m+k+l=\alpha\\
}} P_{m, k}\left[2^{2 m+k} R_{m}\left(\Gamma_{1}\right) M^{k+2 l}\left(\Gamma_{2}\right)\left(2 \delta_{\Gamma_{1}}\right)^{k}\right] .
\end{aligned}
$$


Subsequent sum includes those edges from $R\left(\Gamma_{1}\right)$ whose one end vertex is in $V\left(\Gamma_{1}\right)$ while the other is in $V\left(R\left(\Gamma_{1}\right)\right)-V\left(\Gamma_{1}\right)$.

$$
\begin{aligned}
\sum 5 & =\sum_{w \in V\left(\Gamma_{2}\right)} \sum_{\substack{v_{1} v_{2} \in E\left(R\left(\Gamma_{1}\right)\right) \\
v_{1} \in V\left(\Gamma_{1}\right) \\
v_{2} \in V\left(R\left(\Gamma_{1}\right)\right)-V\left(\Gamma_{1}\right)}}\left[\mathrm{d}\left(v_{1}, w\right) \mathrm{d}\left(v_{2}, w\right)\right]^{\alpha} \\
& =\sum_{w \in V\left(\Gamma_{2}\right)} \sum_{\substack{v_{1} v_{2} \in E\left(R\left(\Gamma_{1}\right)\right) \\
v_{1} \in V\left(\Gamma_{1}\right) \\
v_{2} \in V\left(R\left(\Gamma_{1}\right)\right)-V\left(\Gamma_{1}\right)}}\left[2\left(\mathrm{~d}_{R\left(\Gamma_{1}\right)}\left(v_{1}\right)+\mathrm{d}_{\Gamma_{2}}(w)\right)\right]^{\alpha} \\
& \sum_{\substack{w \in V\left(\Gamma_{2}\right) \\
v_{1} v_{2} \in E\left(R\left(\Gamma_{1}\right)\right) \\
v_{1} \in V\left(\Gamma_{1}\right) \\
v_{2} \in V\left(R\left(\Gamma_{1}\right)\right)-V\left(\Gamma_{1}\right)}}\left[2\left(2 \mathrm{~d}_{G}\left(v_{1}\right)+\mathrm{d}_{\Gamma_{2}}(w)\right)\right]^{\alpha} \\
= & \sum_{\substack{w \in V\left(\Gamma_{2}\right) \\
v_{1} v_{2} \in E\left(R\left(\Gamma_{1}\right)\right) \\
v_{1} \in V\left(\Gamma_{1}\right) \\
v_{2} \in V\left(R\left(\Gamma_{1}\right)\right)-V\left(\Gamma_{1}\right)}}\left[2 \mathrm{~d}_{\Gamma_{2}}(w)+4 \mathrm{~d}_{G}\left(v_{1}\right)\right]^{\alpha}
\end{aligned}
$$

(using binomial theorem)

$$
\begin{aligned}
& =\sum_{w \in V\left(\Gamma_{2}\right)} \sum_{\substack{v_{1} v_{2} \in E\left(R\left(\Gamma_{1}\right)\right) \\
v_{1} \in V\left(\Gamma_{1}\right) \\
v_{2} \in V\left(R\left(\Gamma_{1}\right)\right)-V\left(\Gamma_{1}\right)}}\left[\sum_{i=0}^{\alpha}\left(\begin{array}{c}
\alpha \\
i
\end{array}\right)\left(2 \mathrm{~d}_{\Gamma_{2}}(w)\right)^{\alpha-i}\left(4 \mathrm{~d}_{G}^{i}\left(v_{1}\right)\right)^{i}\right] \\
& =\sum_{i=0}^{\alpha}\left(\begin{array}{c}
\alpha \\
i
\end{array}\right)\left[2^{(\alpha+i)}\left(\sum_{\substack{v_{1} v_{2} \in E\left(R\left(\Gamma_{1}\right)\right) \\
v_{1} \in V\left(\Gamma_{1}\right) \\
v_{2} \in V\left(R\left(\Gamma_{1}\right)\right)-V\left(\Gamma_{1}\right)}} \mathrm{d}_{G}^{i}\left(v_{1}\right)\right)\left(\sum_{w \in V\left(\Gamma_{2}\right)} \mathrm{d}_{\Gamma_{2}}^{(\alpha-i)}(w)\right)\right] \\
& \geq \sum_{i=0}^{\alpha}\left(\begin{array}{c}
\alpha \\
i
\end{array}\right)\left[2^{(\alpha+i)}\left(\sum_{w \in V\left(\Gamma_{2}\right)} \mathrm{d}_{\Gamma_{2}}^{(\alpha-i)}(w)\right) 2\left(\sum_{v_{1} \in V\left(\Gamma_{1}\right)} \mathrm{d}_{G}^{i}\left(v_{1}\right)\right)\right] \geq \sum_{i=0}^{\alpha}\left(\begin{array}{c}
\alpha \\
i
\end{array}\right)\left[2^{(\alpha+i+1)} M^{(\alpha-i)}\left(\Gamma_{2}\right) M^{i}\left(\Gamma_{1}\right)\right] .
\end{aligned}
$$

Using (22), (25), and (26) in (20), we get

$$
\begin{aligned}
R_{\alpha}\left(\Gamma_{1}+{ }_{R} \Gamma_{2}\right) \geq & \sum_{\substack{m, k, l \\
m+k+l=\alpha}} P_{m, k, l} 2^{2 m+k}\left[M^{2 m+k}\left(\Gamma_{1}\right) \chi_{k}\left(\Gamma_{2}\right)\left(\delta_{\Gamma_{2}}\right)^{2 l}+R_{m}\left(\Gamma_{1}\right) M^{k+2 l}\left(\Gamma_{2}\right)\left(2 \delta_{\Gamma_{1}}\right)\right]^{k} \\
& +\sum_{i=0}^{\alpha}\left(\begin{array}{c}
\alpha \\
i
\end{array}\right)\left[2^{(\alpha+i+1)} M^{i}\left(\Gamma_{1}\right) M^{(\alpha-i)}\left(\Gamma_{2}\right)\right]=L B_{R}, R_{\alpha}\left(\Gamma_{1}+{ }_{R} \Gamma_{2}\right) \\
\leq & \sum_{\substack{m, k, l \\
m+k+l=\alpha}} P_{m, k, l} 2^{2 m+k}\left[M^{2 m+k}\left(\Gamma_{1}\right) \chi_{k}\left(\Gamma_{2}\right)\left(\Delta_{\Gamma_{2}}\right)^{2 l}+R_{m}\left(\Gamma_{1}\right) M^{k+2 l}\left(\Gamma_{2}\right)\left(2 \Delta_{\Gamma_{1}}\right)^{k}\right] \\
& +\sum_{i=0}^{\alpha}\left(\begin{array}{c}
\alpha \\
i
\end{array}\right)\left[2^{(\alpha+i+1)} M^{i}\left(\Gamma_{1}\right) M^{(\alpha-i)}\left(\Gamma_{2}\right)\right]=U B_{R} .
\end{aligned}
$$


Equality holds if and only if $\Gamma_{1}$ and $\Gamma_{2}$ are regular graphs with same regularity. This completes the proof.

Example 2. Let $\Gamma_{1}=P_{5}, \Gamma_{2}=P_{4}, \alpha=2$, and $F=R$. Then, $n_{1}=M^{0}\left(P_{5}\right)=5, \quad n_{2}=M^{0}\left(P_{4}\right)=4, \quad e_{1}=\chi_{0}\left(P_{5}\right)=4$,
$e_{2}=\chi_{0}\left(P_{4}\right)=3, \delta_{P_{5}}=1, \Delta_{P_{5}}=2, \delta_{P_{4}}=1$, and $\Delta_{P_{4}}=2$. Now, we compute lower and upper bound of GRI using formulas derived in Theorem 3.

$$
\begin{aligned}
L B_{R}\left(P_{5}+{ }_{R} P_{4}\right)= & \sum_{i=0}^{2}\left(\begin{array}{c}
2 \\
i
\end{array}\right)\left[2^{(3+i)} M^{i}\left(P_{5}\right) M^{(2-i)}\left(P_{4}\right)\right]+\sum_{\substack{m, k, l \\
m+k+l=2}} P_{m, k, l} 2^{2 m+k} \\
& {\left[M^{2 m+k}\left(P_{5}\right) \chi_{k}\left(P_{4}\right)\left(\delta_{P_{4}}\right)^{2 l}+R_{m}\left(P_{5}\right) M^{k+2 l}\left(P_{4}\right)\left(2 \delta_{P_{5}}\right)\right]^{k} } \\
= & 8 M^{0}\left(P_{5}\right) M^{2}\left(P_{4}\right)+32 M\left(P_{5}\right) M\left(P_{4}\right)+32 M^{2}\left(P_{5}\right) M^{0}\left(P_{4}\right)+8 P_{1,1,0}\left(M^{3}\left(P_{5}\right) \chi\left(P_{4}\right)+2 R\left(P_{5}\right) M\left(P_{4}\right)\right) \\
& +2 P_{1,0,1}\left(M^{2}\left(P_{5}\right) \chi_{0}\left(P_{4}\right)+4 R_{0}\left(P_{5}\right) M^{2}\left(P_{4}\right)\right)+2 P_{0,1,1}\left(4 M\left(P_{5}\right) \chi\left(P_{4}\right)+2 R_{0}\left(P_{5}\right) M^{3}\left(P_{4}\right)\right) \\
& +16 P_{2,0,0}\left(M^{4}\left(P_{5}\right) \chi_{0}\left(P_{4}\right)+R_{2}\left(P_{5}\right) M^{0}\left(P_{4}\right)\right)+4 P_{0,2,0}\left(M^{2}\left(P_{5}\right) \chi_{2}\left(P_{4}\right)+4 R_{0}\left(P_{5}\right) M^{2}\left(P_{4}\right)\right) \\
& +P_{0,0,2}\left(16 M^{0}\left(P_{5}\right) \chi_{0}\left(P_{4}\right)+R_{0}\left(P_{5}\right) M^{4}\left(P_{4}\right)\right), \\
& 8(5)(10)+32(8)(10)+32(14)(4)+16(26(10)+12(6)(2))+8(14(3)(4)+12(10)) \\
& +4(8(10)(4)+4(18)(2))+16(50(3)+40(4))+4(14(34)+4(10)(4))+(5(3)(16)+4(34))=23256 .
\end{aligned}
$$

In similar way, we calculate $U B_{R}\left(P_{5}+{ }_{R} P_{4}\right)=28056$.

To compute exact value for $R_{2}\left(P_{5}+{ }_{R} P_{4}\right)$, we need edge partition of graph $P_{5}+{ }_{R} P_{4}$, which is given in Table 4 .

Exact value of GRI of $P_{5}+{ }_{R} P_{4}$ for $\alpha=2$ is given by $R_{2}\left(P_{5}+{ }_{R} P_{4}\right)=\sum_{v w \in E\left(P_{5}+{ }_{R} P_{4}\right)}\left(\mathrm{d}_{P_{5}}(v) \mathrm{d}_{P_{4}}(w)\right)^{2}=24592$.

Clearly,

$$
23256 \leq R_{2}\left(P_{5}+{ }_{R} P_{4}\right)=24592 \leq 28056 .
$$

Moreover, we computed actual values along with corresponding bounds of GRI for various cases, and some are presented in Table 5.

Theorem 4. Let $\Gamma_{1}$ and $\Gamma_{2}$ be two simple, finite, and connected graphs. For $\alpha \in \mathcal{N}$, the general Randić index of Q-sum graph is $L B_{Q} \leq R_{\alpha}\left(\Gamma_{1}+_{Q} \Gamma_{2}\right) \leq U B_{Q}$, where

$$
\begin{aligned}
L B_{Q} & =\sum_{\substack{m, k, l \\
m+k+l=\alpha}} P_{m, k, l}\left[M^{2 m+k}\left(\Gamma_{1}\right) R_{l}\left(\Gamma_{2}\right)\left(2 \delta_{\Gamma_{2}}\right)^{k}\right]+\sum_{i=0}^{\alpha}\left(\begin{array}{c}
\alpha \\
i
\end{array}\right)\left[M^{i}\left(\Gamma_{2}\right) \chi_{\alpha}\left(\Gamma_{1}\right)\left(\delta_{\Gamma_{1}}\right)^{\alpha-i}\right]+n_{2} \chi_{\alpha}\left(\Gamma_{1}\right)\left(2 \delta_{\Gamma_{1}}\right)^{\alpha} \\
U B_{Q} & =\sum_{\substack{m, k, l \\
m+k+l=\alpha}} P_{m, k, l}\left[M^{2 m+k}\left(\Gamma_{1}\right) R_{l}\left(\Gamma_{2}\right)\left(2 \Delta_{\Gamma_{2}}\right)^{k}\right]+\sum_{i=0}^{\alpha}\left(\begin{array}{c}
\alpha \\
i
\end{array}\right)\left[M^{i}\left(\Gamma_{2}\right) \chi_{\alpha}\left(\Gamma_{1}\right)\left(\Delta_{\Gamma_{1}}\right)^{\alpha-i}\right]+n_{2} \chi_{\alpha}\left(\Gamma_{1}\right)\left(2 \Delta_{\Gamma_{1}}\right)^{\alpha}
\end{aligned}
$$

and $P_{m, k, l}=(m+k+l) ! / m ! k ! l !$. Equality holds if and only if $\Gamma_{1}$ and $\Gamma_{2}$ are regular graphs with same regularity.
Proof. Suppose that $\mathrm{d}(v, w)=\mathrm{d}_{\left(\Gamma_{1}+{ }_{Q} \Gamma_{2}\right)}(v, w)$ denotes the degree of vertex $(v, w)$ in the $Q$-sum graph $\Gamma_{1}+{ }_{Q} \Gamma_{2}$. Then general Randić index for $Q$-sum graph is calculated as 
TABlE 4: Edge partition based upon degree of end vertices of graph $P_{5}+{ }_{R} P_{4}$.

\begin{tabular}{lcccccccccccc}
\hline$\left(d_{v}, d_{w}\right)$ & $(2,3)$ & $(2,4)$ & $(2,5)$ & $(2,6)$ & $(3,4)$ & $(3,5)$ & $(4,4)$ & $(4,6)$ & $(5,5)$ & $(5,6)$ & $(6,6)$ \\
\hline$|E|$ & 4 & 4 & 12 & 12 & 4 & 4 & 2 & 4 & 4 & 6 & 7 \\
\hline
\end{tabular}

TABLE 5: Few more cases of lower and upper bounds regarding $R_{\alpha}\left(\Gamma_{1}+{ }_{R} \Gamma_{2}\right)$.

\begin{tabular}{lllc}
\hline$\Gamma_{1}$ & $\Gamma_{2}$ & $\alpha$ & $L B_{R} \leq R_{\alpha}\left(\Gamma_{1}+{ }_{R} \Gamma_{2}\right) \leq U B_{R}$ \\
\hline$P_{4}$ & $C_{3}$ & 2 & $15696 \leq 18768 \leq 19248$ \\
$P_{4}$ & $C_{3}$ & 3 & $425952 \leq 551232 \leq 589632$ \\
$P_{5}$ & $P_{4}$ & 3 & $526448 \leq 698288 \leq 699904$ \\
\hline
\end{tabular}

$$
\begin{aligned}
& R_{\alpha}\left(\Gamma_{1}+{ }_{Q} \Gamma_{2}\right)=\sum_{\left(v_{1}, w_{1}\right)} \sum_{\left(v_{2}, w_{2}\right) \in E\left(\Gamma_{1}+{ }_{Q} \Gamma_{2}\right)}\left[\mathrm{d}\left(v_{1}, w_{1}\right) \mathrm{d}\left(v_{2}, w_{2}\right)\right]^{\alpha}=\sum_{v \in V\left(\Gamma_{1}\right)} \sum_{w_{1} w_{2} \in E\left(\Gamma_{2}\right)}\left[\mathrm{d}\left(v, w_{1}\right) \mathrm{d}\left(v, w_{2}\right)\right]^{\alpha} \\
& +\sum_{w \in V\left(\Gamma_{2}\right)} \sum_{v_{1} v_{2} \in E\left(Q\left(\Gamma_{1}\right)\right)}\left[\mathrm{d}\left(v_{1}, w\right) \mathrm{d}\left(v_{2}, w\right)\right]^{\alpha} \\
& =\sum_{v \in V\left(\Gamma_{1}\right)} \sum_{w_{1} w_{2} \in E\left(\Gamma_{2}\right)}\left[\mathrm{d}\left(v, w_{1}\right) \mathrm{d}\left(v, w_{2}\right)\right]+\sum_{w \in V\left(\Gamma_{2}\right)} \sum_{v_{1} v_{2} \in E\left(Q\left(\Gamma_{1}\right)\right)}\left[\mathrm{d}\left(v_{1}, w\right) \mathrm{d}\left(v_{2}, w\right)\right]^{\alpha} \\
& +\sum_{w \in V\left(\Gamma_{2}\right)} \sum_{\substack{v_{1} v_{2} \in E\left(Q\left(\Gamma_{1}\right)\right) \\
v_{1} v_{2} \in V\left(Q\left(\Gamma_{1}\right)\right)-V\left(\Gamma_{1}\right)}}\left[\mathrm{d}\left(v_{1}, w\right) \mathrm{d}\left(v_{2}, w\right)\right]^{\alpha \alpha} \begin{array}{c}
v_{1} \in V\left(\Gamma_{1}\right) \\
v_{2} \in V\left(Q\left(\Gamma_{1}\right)\right)-V\left(\Gamma_{1}\right)
\end{array} \\
& =\sum 6+\sum 7+\sum 8, \\
& \sum 6=\sum_{v \in V\left(\Gamma_{1}\right)} \sum_{w_{1} w_{2} \in E\left(\Gamma_{2}\right)}\left[\mathrm{d}\left(v, w_{1}\right) \mathrm{d}\left(v, w_{2}\right)\right]^{\alpha}=\sum_{v \in V\left(\Gamma_{1}\right)} \sum_{w_{1} w_{2} \in E\left(\Gamma_{2}\right)}\left[\left(\mathrm{d}_{Q\left(\Gamma_{1}\right)}(v)+\mathrm{d}_{\Gamma_{2}}\left(w_{1}\right)\right)\left(\mathrm{d}_{Q\left(\Gamma_{1}\right)}(v)+\mathrm{d}_{\Gamma_{2}}\left(w_{2}\right)\right)\right]^{\alpha} \\
& =\sum_{v \in V\left(\Gamma_{1}\right)} \sum_{w_{1} w_{2} \in E\left(\Gamma_{2}\right)}\left[\left(\mathrm{d}_{\Gamma_{1}}(v)+\mathrm{d}_{\Gamma_{2}}\left(w_{1}\right)\right)\left(\mathrm{d}_{\Gamma_{1}}(v)+\mathrm{d}_{\Gamma_{2}}\left(w_{1}\right)\right)\right]^{\alpha}, \\
& \sum 6=\sum_{v \in V\left(\Gamma_{1}\right)} \sum_{w_{1} w_{2} \in E\left(\Gamma_{2}\right)}\left[\mathrm{d}_{\Gamma_{1}}^{2}(v)+\mathrm{d}_{\Gamma_{1}}(v)\left(\mathrm{d}_{\Gamma_{2}}\left(w_{1}\right)+\mathrm{d}_{\Gamma_{2}}\left(w_{2}\right)\right)+\mathrm{d}_{\Gamma_{2}}\left(w_{1}\right) \mathrm{d}_{\Gamma_{2}}\left(w_{2}\right)^{\alpha}\right] .
\end{aligned}
$$

Applying trinomial theorem, we get

$$
\sum 6=\sum_{v \in V\left(\Gamma_{1}\right)} \sum_{w_{1} w_{2} \in E\left(\Gamma_{2}\right)}\left[\sum_{\substack{m, k, l \\ m+k+l=\alpha}} P_{m, k, l} \mathrm{~d}_{\Gamma_{1}}^{2 m}(v) \mathrm{d}_{\Gamma_{1}}^{k}(v)\left(\mathrm{d}_{\Gamma_{2}}\left(w_{1}\right)+\mathrm{d}_{\Gamma_{2}}\left(w_{2}\right)\right)^{k}\left(\mathrm{~d}_{\Gamma_{2}}\left(w_{1}\right) \mathrm{d}_{\Gamma_{2}}\left(w_{2}\right)\right)^{l}\right],
$$


where $P_{m, k, l}=(m+k+l) ! / m ! k ! l !$.

$$
\begin{aligned}
\sum 6= & \sum_{\substack{m, k, l \\
m+k+l=\alpha}} P_{m, k, l}\left[\sum_{v \in V\left(\Gamma_{1}\right)} \sum_{w_{1} w_{2} \in E\left(\Gamma_{2}\right)}\left(\left(d_{\Gamma_{2}}\left(w_{1}\right)+d_{\Gamma_{2}}\left(w_{2}\right)\right)^{k} d_{\Gamma_{1}}^{2 m}(v) d_{\Gamma_{1}}^{k}(v)\left(d_{\Gamma_{2}}\left(w_{1}\right) d_{\Gamma_{2}}\left(w_{2}\right)\right)^{l}\right)\right] \\
= & \left.\sum_{\substack{m, k, l \\
m+k+l=\alpha}} P_{m, k, l}\left[\sum_{w_{1} w_{2} \in E\left(\Gamma_{2}\right)}\left(d_{\Gamma_{2}}\left(w_{1}\right) d_{\Gamma_{2}}\left(w_{2}\right)\right)^{l}\left(d_{\Gamma_{2}} w_{1}\right)+d_{\Gamma_{2}}\left(w_{2}\right)\right)^{k}\right]\left[\sum_{v \in V\left(\Gamma_{1}\right)} d_{\Gamma_{1}}^{2 m+k}(v)\right] \geq \\
& \sum_{\substack{m, k, l \\
m+k+l=\alpha}} P_{m, k, l}\left[M^{2 m+k}\left(\Gamma_{1}\right) R_{l}\left(\Gamma_{2}\right)\left(2 \delta_{\Gamma_{2}}\right)^{k}\right] .
\end{aligned}
$$

$\sum 7$ involves those edges from $Q\left(\Gamma_{1}\right)$ whose one end vertex is in $V\left(\Gamma_{1}\right)$ and the other is in $V\left(Q\left(\Gamma_{1}\right)\right)-V\left(\Gamma_{1}\right)$.

$$
\begin{aligned}
\sum 7 & =\sum_{w \in V\left(\Gamma_{2}\right)} \sum_{\substack{v_{1} v_{2} \in E\left(Q\left(\Gamma_{1}\right)\right) \\
v_{1} \in V\left(\Gamma_{1}\right) \\
v_{2} \in V\left(Q\left(\Gamma_{1}\right)\right)-V\left(\Gamma_{1}\right)}}\left[d\left(v_{1}, w\right) d\left(v_{2}, w\right)\right]^{\alpha} \\
& \sum_{w \in V\left(\Gamma_{2}\right)} \sum_{\substack{v_{1} v_{2} \in E\left(Q\left(\Gamma_{1}\right)\right) \\
v_{1} \in V\left(\Gamma_{1}\right) \\
v_{2} \in V\left(Q\left(\Gamma_{1}\right)\right)-V\left(\Gamma_{1}\right)}}\left[\left(d_{Q\left(\Gamma_{1}\right)}\left(v_{1}\right)+d_{\Gamma_{2}}(w)\right) d_{Q\left(\Gamma_{1}\right)}\left(v_{2}\right)\right]^{\alpha} \\
& \left.\sum_{w \in V\left(\Gamma_{2}\right)} \sum_{\substack{v_{1} v_{2} \in E\left(Q\left(\Gamma_{1}\right)\right) \\
v_{1} \in V\left(\Gamma_{1}\right) \\
v_{2} \in V\left(Q\left(\Gamma_{1}\right)\right)-V\left(\Gamma_{1}\right)}}\left[\left(d_{\Gamma_{1}} v_{1}\right)+d_{\Gamma_{2}}(w)\right)^{\alpha}\left(d_{Q\left(\Gamma_{1}\right)}\left(v_{2}\right)\right)^{\alpha}\right] .
\end{aligned}
$$

Now applying binomial theorem, we have

$$
\begin{aligned}
\sum 7 & =\sum_{w \in V\left(\Gamma_{2}\right)} \sum_{\substack{v_{1} v_{2} \in E\left(Q\left(\Gamma_{1}\right)\right) \\
v_{1} \in V\left(\Gamma_{1}\right) \\
v_{2} \in V\left(Q\left(\Gamma_{1}\right)\right)-V\left(\Gamma_{1}\right)}}\left[\sum_{i=0}^{\alpha}\left(\begin{array}{c}
\alpha \\
i
\end{array}\right)\left(\mathrm{d}_{\Gamma_{1}}\left(v_{1}\right)\right)^{\alpha-i}\left(\mathrm{~d}_{\Gamma_{2}}(w)\right)^{i}\left(\mathrm{~d}_{Q\left(\Gamma_{1}\right)}\left(v_{2}\right)\right)\right]^{\alpha} \\
& =\sum_{i=0}^{\alpha}\left(\begin{array}{c}
\alpha \\
i
\end{array}\right)\left(\sum_{\substack{v_{1} v_{2} \in E\left(Q\left(\Gamma_{1}\right)\right) \\
v_{1} \in V\left(\Gamma_{1}\right) \\
v_{2} \in V\left(Q\left(\Gamma_{1}\right)\right)-V\left(\Gamma_{1}\right)}} \mathrm{d}_{Q\left(\Gamma_{1}\right)}^{\alpha}\left(v_{2}\right)\left(\sum_{w \in V\left(\Gamma_{2}\right)} \mathrm{d}_{\Gamma_{2}}^{i}(w)\right)\left(\mathrm{d}_{\Gamma_{1}}^{\alpha-i}\left(v_{1}\right)\right)\right.
\end{aligned}
$$


It can easily be observed that $\mathrm{d}_{Q\left(\Gamma_{1}\right)}\left(v_{2}\right)=\left(\mathrm{d}_{\Gamma_{1}}\left(u_{s}\right)+\right.$ $\left.\mathrm{d}_{\Gamma_{1}}\left(u_{t}\right)\right)$ for $v_{2} \in V\left(Q\left(\Gamma_{1}\right)\right)-V\left(\Gamma_{1}\right)$, where $v_{2}$ is the vertex inserted into the edge $u_{s} u_{t} \in E\left(\Gamma_{1}\right)$. In addition,

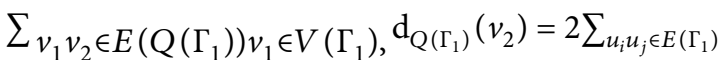

$v_{2} \in V\left(Q\left(\Gamma_{1}\right)\right)-V\left(\Gamma_{1}\right)$

$\left(\mathrm{d}_{\Gamma_{1}}\left(u_{i}\right)+\mathrm{d}_{\Gamma_{1}}\left(u_{j}\right)\right)$.
Also, using the fact $\delta_{\Gamma_{1}}(v) \leq d_{\Gamma_{1}}(v) \forall v \in V\left(\Gamma_{1}\right)$, we have

$$
\sum 7 \geq \sum_{i=0}^{\alpha}\left(\begin{array}{c}
\alpha \\
i
\end{array}\right)\left[2\left(\sum_{u_{i} u_{j} \in E\left(\Gamma_{1}\right)}\left(\mathrm{d}_{\Gamma_{1}}\left(u_{i}\right)+\mathrm{d}_{\Gamma_{1}}\left(u_{j}\right)\right)^{\alpha}\right) M^{i}\left(\Gamma_{2}\right)\left(\delta_{\Gamma_{1}}\right)^{\alpha-i}\right]=\sum_{i=0}^{\alpha}\left(\begin{array}{c}
\alpha \\
i
\end{array}\right)\left[2 M^{i}\left(\Gamma_{2}\right) \chi_{\alpha}\left(\Gamma_{1}\right)\left(\delta_{\Gamma_{1}}\right)^{\alpha-i}\right] .
$$

Next sum contains those edges from $Q\left(\Gamma_{1}\right)$ whose both end vertices are in $V\left(Q\left(\Gamma_{1}\right)\right)-V\left(\Gamma_{1}\right)$.

$$
\begin{aligned}
\sum 8 & =\sum_{w \in V\left(\Gamma_{2}\right)} \sum_{\substack{v_{1} v_{2} \in E\left(Q\left(\Gamma_{1}\right)\right) v_{1}, v_{2} \in V\left(Q\left(\Gamma_{1}\right)\right)-V\left(\Gamma_{1}\right)}}\left[\mathrm{d}\left(v_{1}, w\right) \mathrm{d}\left(v_{2}, w\right)\right]^{\alpha}=\sum_{w \in V\left(\Gamma_{2}\right)} \sum_{\substack{v_{1} v_{2} \in E\left(Q\left(\Gamma_{1}\right)\right) v_{1}, v_{2} \in V\left(Q\left(\Gamma_{1}\right)\right)-V\left(\Gamma_{1}\right)}}\left[\mathrm{d}_{Q\left(\Gamma_{1}\right)}\left(v_{1}\right) \mathrm{d}_{Q\left(\Gamma_{1}\right)}\left(v_{2}\right)\right]^{\alpha} \\
& =\sum_{w \in V\left(\Gamma_{2}\right)} \sum_{\substack{u_{s} u_{t} \in E\left(\Gamma_{1}\right) \\
u_{t} u_{r} \in E\left(\Gamma_{1}\right)}}\left[\left(\mathrm{d}_{\Gamma_{1}}\left(u_{s}\right)+\mathrm{d}_{\Gamma_{1}}\left(u_{t}\right)\right)\left(\mathrm{d}_{\Gamma_{1}}\left(u_{t}\right)+\mathrm{d}_{\Gamma_{1}}\left(u_{r}\right)\right)\right]^{\alpha},
\end{aligned}
$$

where $v_{1}$ and $v_{2}$ are the vertices embedded into the edges $u_{s} u_{t}$ and $u_{t} u_{r}$ of $\Gamma_{1}$, respectively.

$$
\sum 8=\sum_{w \in V\left(\Gamma_{2}\right)} \sum_{u_{s} u_{t} \in E\left(\Gamma_{1}\right)}\left[\left(\mathrm{d}_{\Gamma_{1}}\left(u_{s}\right)+\mathrm{d}_{\Gamma_{1}}\left(u_{t}\right)\right)^{\alpha}\left(\mathrm{d}_{\Gamma_{1}}\left(u_{t}\right)+\mathrm{d}_{\Gamma_{1}}\left(u_{r}\right)\right)^{\alpha}\right] \geq n_{2}\left[\sum_{u_{s} u_{t} \in E\left(\Gamma_{1}\right)}\left(\mathrm{d}_{\Gamma_{1}}\left(u_{s}\right)+\mathrm{d}_{\Gamma_{1}}\left(u_{t}\right)\right)^{\alpha}\left(2 \delta_{\Gamma_{1}}\right)^{\alpha}\right]=n_{2} \chi_{\alpha}\left(\Gamma_{1}\right)\left(2 \delta_{\Gamma_{1}}\right)^{\alpha}
$$

Using equations (34)-(39) in (31), we get

$$
R_{\alpha}\left(\Gamma_{1}+{ }_{Q} \Gamma_{2}\right) \geq \sum_{\substack{m, k, l \\
m+k+l=\alpha}} P_{m, k, l}\left[M^{2 m+k}\left(\Gamma_{1}\right) R_{l}\left(\Gamma_{2}\right)\left(2 \delta_{\Gamma_{2}}\right)^{k}\right]+\sum_{i=0}^{\alpha}\left(\begin{array}{c}
\alpha \\
i
\end{array}\right)\left[2 M^{i}\left(\Gamma_{2}\right) \chi_{\alpha}\left(\Gamma_{1}\right)\left(\delta_{\Gamma_{1}}\right)^{\alpha-i}\right]+n_{2} \chi_{\alpha}\left(\Gamma_{1}\right)\left(2 \delta_{\Gamma_{1}}\right)^{\alpha}=L B_{Q} .
$$

Similarly, we have

$$
R_{\alpha}\left(\Gamma_{1}+{ }_{Q} \Gamma_{2}\right) \leq \sum_{\substack{m, k, l \\
m+k+l=\alpha}} P_{m, k, l}\left[M^{2 m+k}\left(\Gamma_{1}\right) R_{l}\left(\Gamma_{2}\right)\left(2 \Delta_{\Gamma_{2}}\right)^{k}\right]+\sum_{i=0}^{\alpha}\left(\begin{array}{c}
\alpha \\
i
\end{array}\right)\left[2 M^{i}\left(\Gamma_{2}\right) \chi_{\alpha}\left(\Gamma_{1}\right)\left(\Delta_{\Gamma_{1}}\right)^{\alpha-i}\right]+n_{2} \chi_{\alpha}\left(\Gamma_{1}\right)\left(2 \Delta_{\Gamma_{1}}\right)^{\alpha}=U B_{Q}
$$


Equality holds if and only if $\Gamma_{1}$ and $\Gamma_{2}$ are regular graphs with same regularity. This completes the proof.
Example 3. To compute lower and upper bound of GRI using formulas derived in Theorem 4 for the graphs and corresponding parameters presented in Example 2. we get

$$
\begin{aligned}
U B_{Q}\left(P_{5}{ }_{Q} P_{4}\right)= & \sum_{\substack{m, k, l \\
m+k+l=2}} P_{m, k, l}\left[M^{2 m+k}\left(P_{5}\right) R_{l}\left(P_{4}\right)\left(2 \Delta_{P_{4}}\right)^{k}\right]+\sum_{i=0}^{2}\left(\begin{array}{c}
2 \\
i
\end{array}\right)\left[2 M^{i}\left(P_{4}\right) \chi_{\alpha}\left(P_{5}\right)\left(\Delta_{P_{5}}\right)^{\alpha-i}\right]+n_{2} \chi_{\alpha}\left(P_{5}\right)\left(2 \Delta_{P_{5}}\right)^{\alpha}, P^{2} \\
U B_{Q}\left(P_{5}+{ }_{Q} P_{4}\right)= & P_{2,0,0}\left(M^{4}\left(P_{5}\right) R_{0}\left(P_{4}\right)\right)+P_{0,2,0}\left(M^{2}\left(P_{5}\right) R_{0}\left(P_{4}\right)(16)\right)+P_{0,0,2}\left(M^{0}\left(P_{5}\right) R_{2}\left(P_{4}\right)\right)+P_{1,1,0}\left(M^{3}\left(P_{5}\right) R_{0}\left(P_{4}\right)(4)\right) \\
& +P_{1,0,1}\left(M^{2}\left(P_{5}\right) R\left(P_{4}\right)\right)+P_{0,1,1}\left(M\left(P_{5}\right) R\left(P_{4}\right)(4)\right)+2 \chi_{2}\left(P_{5}\right)\left(4 M^{0}\left(P_{4}\right)+4 M\left(P_{4}\right)+M^{2}\left(P_{4}\right)\right)+64 \chi_{2}\left(P_{5}\right) \\
= & (50(3)+14(3)(16)+5(24)) \\
& +2(26(3)(4)+14(8)+8(8)(4))+100(4(4)+2(6)(2)+10)+64(50)=10502 .
\end{aligned}
$$

$$
4630 \leq R_{2}\left(P_{5}+{ }_{Q} P_{4}\right)=8682 \leq 10502 .
$$

In similar way, we calculate $L B_{Q}\left(P_{5}{ }^{+} P_{4}\right)=4630$.

To compute exact value for $R_{2}\left(P_{5}+{ }_{Q} P_{4}\right)$, we need edge partition of graph $P_{5}{ }^{+} P_{4}$, and observe 8 edges with end vertex degrees $(2,3), 10$ edges with end vertex degrees $(3,3)$, 26 edges with end vertex degrees $(3,4)$, and 15 edges with end vertex degrees $(4,4)$, respectively.

Now, we compute exact value of GRI of $P_{5}{ }^{+}{ }_{Q} P_{4}$ for $\alpha=2 . R_{2}\left(P_{5}{ }_{Q} P_{4}\right)=\sum_{v w \in E\left(P_{5}+{ }_{Q} P_{4}\right)}\left(\mathrm{d}_{P_{5}}(v) \mathrm{d}_{P_{4}}(w)\right)^{2}=8682$.

Obviously,
Moreover, we computed actual values along with corresponding bounds of GRI for various cases, and some are presented in Table 6 .

Theorem 5. Let $\Gamma_{1}$ and $\Gamma_{2}$ be two simple, finite, and connected graphs. For $\alpha \in \mathcal{N}$, the general Randić index of T-sum graph is $L B_{T} \leq R_{\alpha}\left(\Gamma_{1}+{ }_{T} \Gamma_{2}\right) \leq U B_{T}$, where

$$
\begin{aligned}
L B_{T}= & \sum_{\substack{m, k, l \\
m+k+l=\alpha}} P_{m, k, l}\left[M^{2 m+k}\left(\Gamma_{1}\right) \chi_{k}\left(\Gamma_{2}\right)\left(\delta_{\Gamma_{2}}\right)^{2 l}+2^{2 m+k} R_{m}\left(\Gamma_{1}\right) M^{k+2 l}\left(\Gamma_{2}\right)\left(2 \delta_{\Gamma_{1}}\right)^{k}\right]+\sum_{i=0}^{\alpha}\left(\begin{array}{c}
\alpha \\
i
\end{array}\right)\left[2 M^{i}\left(\Gamma_{2}\right) \chi_{\alpha}\left(\Gamma_{1}\right)\left(\delta_{\Gamma_{1}}\right)^{\alpha-i}\right] \\
& +n_{2} \chi_{\alpha}\left(\Gamma_{1}\right)\left(2 \delta_{\Gamma_{1}}\right)^{\alpha}, \\
U B_{T}= & \sum_{\substack{m, k, l \\
m+k+l=\alpha}} P_{m, k, l}\left[M^{2 m+k}\left(\Gamma_{1}\right) \chi_{k}\left(\Gamma_{2}\right)\left(\Delta_{\Gamma_{2}}\right)^{2 l}+2^{2 m+k} R_{m}\left(\Gamma_{1}\right) M^{k+2 l}\left(\Gamma_{2}\right)\left(2 \Delta_{\Gamma_{1}}\right)^{k}\right]+\sum_{i=0}^{\alpha}\left(\begin{array}{c}
\alpha \\
i
\end{array}\right)\left[2 M^{i}\left(\Gamma_{2}\right) \chi_{\alpha}\left(\Gamma_{1}\right)\left(\Delta_{\Gamma_{1}}\right)^{\alpha-i}\right] \\
& +n_{2} \chi_{\alpha}\left(\Gamma_{1}\right)\left(2 \Delta_{\Gamma_{1}}\right)^{\alpha},
\end{aligned}
$$


TABLE 6: Few more cases of lower and upper bounds regarding $R_{\alpha}\left(\Gamma_{1}+{ }_{Q} \Gamma_{2}\right)$.

\begin{tabular}{lllc}
\hline$\Gamma_{1}$ & $\Gamma_{2}$ & $\alpha$ & $L B_{Q} \leq R_{\alpha}\left(\Gamma_{1}+\Gamma_{Q}\right) \leq U B_{Q}$ \\
\hline$P_{4}$ & $C_{3}$ & 2 & $4266 \leq 5772 \leq 6918$ \\
$P_{4}$ & $C_{3}$ & 3 & $50898 \leq 78636 \leq 96918$ \\
$P_{5}$ & $P_{4}$ & 3 & $43674 \leq 115386 \leq 144422$ \\
\hline
\end{tabular}

and $P_{m, k, l}=(m+k+l) ! / m ! k ! l !$. Equality holds if and only if $\Gamma_{1}$ and $\Gamma_{2}$ are regular graphs with same regularity.

Proof. In total graph, we know $\mathrm{d}_{\Gamma_{1}+{ }_{T} \Gamma_{2}}(v, w)=\mathrm{d}_{\Gamma_{1}+{ }_{R} \Gamma_{2}}(v, w)$ for $\quad v \in V\left(\Gamma_{1}\right) \quad$ and $w \in V\left(\Gamma_{2}\right)$ and $\mathrm{d}_{\Gamma_{1}+T_{T} \Gamma_{2}}(v, w)=\mathrm{d}_{\Gamma_{1}{ }_{Q} \Gamma_{2}}(v, w)$ for $v \in V\left(T\left(\Gamma_{1}\right)\right)-V\left(\Gamma_{1}\right)$ and $w \in V\left(\Gamma_{2}\right)$. The lower and upper for total graph can be attained as a direct consequence of Theorems 3 and 4 . Moreover, the results and examples illustrated in Theorems 3 and 4 implicate that the bounds provided in Theorem 5 perform significantly well, and hence examples are omitted.
Now, we propose lower and upper bounds of general Randić index of $F$-sum graphs for $\alpha \in \mathscr{R}$ and following results are intimately tied with generalized binomial and trinomial theorems.

\section{Applications}

4.1. Results for Cycles $C_{a}$ and $C_{b}$. Let $C_{a}$ and $C_{b}$ be two cycle graphs with vertices $a$ and $b$, respectively. Then, using Theorems 2-5, the lower and upper bounds of general Randić index of $F$-sum graphs $C_{a}+{ }_{S} C_{b}, C_{a}+{ }_{R} C_{b}, C_{a}+{ }_{Q} C_{b}$, and $C_{a}+{ }_{T} C_{b}$, are given as follows:

$$
\begin{aligned}
& L B_{S}=U B_{S}=\sum_{\substack{m, k, l \\
m+k+l=\alpha}} P_{m, k, l}\left[a b 2^{2 m+3 k+2 l}\right]+\sum_{i=0}^{\alpha}\left(\begin{array}{c}
\alpha \\
i
\end{array}\right) b^{2} 2^{2 \alpha+1}, \\
& L B_{R}=U B_{R}=\sum_{i=0}^{\alpha}\left(\begin{array}{c}
\alpha \\
i
\end{array}\right) a b 2^{2 \alpha+i-1}+\sum_{\substack{m, k, l \\
m+k+l=\alpha}} P_{m, k, l}\left[a b 2^{2 m+3 k+2 l}+b 2^{m+4 k+2 l+1}\left(1+(a-2) 2^{m-1}\right)\right], \\
& L B_{Q}=U B_{Q}=\sum_{\substack{m, k, l \\
m+k+l=\alpha}} P_{m, k, l}\left[a b 2^{2 m+3 k+2 l}\right]+\sum_{i=0}^{\alpha}\left(\begin{array}{c}
\alpha \\
i
\end{array}\right) a b 2^{3 \alpha+1}+a b 2^{4 \alpha}, \\
& L B_{T}=U B_{T}=\sum_{\substack{m, k, l \\
m+k+l=\alpha}} P_{m, k, l}(a b) 2^{(2 m+4 k)}\left[2^{k}+2^{2 m+2 l}\right]+\sum_{i=0}^{\alpha}\left(\begin{array}{c}
\alpha \\
i
\end{array}\right) a b 2^{3 \alpha+1}+a b 2^{4 \alpha} .
\end{aligned}
$$

Note that lower and upper bounds are equal due to the reason that $C_{a}$ and $C_{b}$ are regular graphs with same regularity.
4.2. Results for Paths $P_{a}$ and $P_{b}$. Let $P_{a}$ and $P_{b}$ be two path graphs with vertices $a$ and $b$, respectively. Then, using Theorems 2-5, the lower and upper bounds of general 
Randić index of $F$-sum graphs $P_{a}+{ }_{S} P_{b}, P_{a}+{ }_{R} P_{b}, P_{a}+_{Q} P_{b}$, and $P_{a}+{ }_{T} P_{b}$, are given as

$$
\begin{aligned}
& L B_{S}=\sum_{\substack{m, k, l \\
m+k+l=\alpha}} P_{m, k, l}\left[2^{(k+l)}\left(2+(a-2) 2^{2 m+k}\right)\left(2+(b-2) 2^{l}\right)\right]+\left[\left(\sum_{i=0}^{\alpha}\left(\begin{array}{c}
\alpha \\
i
\end{array}\right)\left(4+(b-2) 2^{i+1}\right)\right)+b 2^{\alpha}\right]\left(2(3)^{\alpha}+(a-2) 2^{2 \alpha}\right), \\
& U B_{S}=\sum_{\substack{m, k, l \\
m+k+l=\alpha}} P_{m, k, l}\left[2^{(k+l)}\left(2+(a-2) 2^{2 m+k}\right)\left(2+(b-2) 2^{l}\right)\right]+\left[\left(\sum_{i=0}^{\alpha}\left(\begin{array}{c}
\alpha \\
i
\end{array}\right) 2^{\alpha-i+1}\left(2+(b-2) 2^{i}\right)\right)+b 2^{2 \alpha}\right]\left(2(3)^{\alpha}+(a-2) 2^{2 \alpha}\right) \\
& L B_{R}=\left[\left(\sum_{i=0}^{\alpha}\left(\begin{array}{c}
\alpha \\
i
\end{array}\right)\left(4+(b-2) 2^{i+1}\right)\right)+2^{3 m+2 k}\left(2+(a-2) 2^{m}\right)\right]+b 2^{\alpha}\left(2(3)^{\alpha}+(a-2) 2^{\alpha}\right)+\sum_{\substack{m, k, l \\
m+k+l=\alpha}} P_{m, k, l}\left[\left(2+(a-2) 2^{2 m+k}\right)\right. \\
& \left.\cdot\left(2(3)^{k}+(b-2) 2^{2 k}\right) 2^{3 m+2 k}+\left(2+(a-2) 2^{m}\right)\left(2+(b-2) 2^{2 k+l}\right)\right] \\
& U B_{R}=\sum_{\substack{m, k, l \\
m+k+l=\alpha}} P_{m, k, l}\left[2^{2 l}\left(2+(a-2) 2^{2 m+k}\right)\left(2(3)^{k}+(b-2) 2^{2 k}\right)+2^{3 m+2 k}\left(2+(a-2) 2^{m}\right)\right. \\
& \left.+2^{3 m+3 k}\left(2+(a-2) 2^{m}\right)\left(2+(b-2) 2^{2 k+l}\right)\right] \\
& +\left[\left(\sum_{i=0}^{\alpha}\left(\begin{array}{c}
\alpha \\
i
\end{array}\right) 2^{\alpha-i+1}\left(2+(b-2) 2^{i}\right)\right)+2^{3 m+2 k}\left(2+(a-2) 2^{m}\right)+b 2^{2 \alpha}\right]\left(2(3)^{\alpha}+(a-2) 2^{\alpha}\right), \\
& L B_{Q}=\sum_{\substack{m, k, l \\
m+k+l=\alpha}} P_{m, k, l}\left[2^{(k+l)}\left(2+(a-2) 2^{2 m+k}\right)\left(2+(b-2) 2^{l}\right)\right]+\left[\left(\sum_{i=0}^{\alpha}\left(\begin{array}{c}
\alpha \\
i
\end{array}\right)\left(4+(b-2) 2^{i+1}\right)\right)+b 2^{\alpha}\right]\left(2(3)^{\alpha}+(a-2) 2^{2 \alpha}\right) \\
& U B_{Q}=\sum_{\substack{m, k, l \\
m+k+l=\alpha}} P_{m, k, l}\left[2^{(k+l)}\left(2+(a-2) 2^{2 m+k}\right)\left(2+(b-2) 2^{l}\right)\right]+\left[\left(\sum_{i=0}^{\alpha}\left(\begin{array}{c}
\alpha \\
i
\end{array}\right) 2^{\alpha-i+1}\left(2+(b-2) 2^{i}\right)\right)+b 2^{2 \alpha}\right] \\
& \cdot\left(2(3)^{\alpha}+(a-2) 2^{2 \alpha}\right) \\
& L B_{T}=\left[\left(\sum_{i=0}^{\alpha}\left(\begin{array}{c}
\alpha \\
i
\end{array}\right)\left(4+(b-2) 2^{i+1}\right)\right)+2^{3 m+2 k}\left(2+(a-2) 2^{m}\right)\right]+b 2^{\alpha}\left(2(3)^{\alpha}+(a-2) 2^{\alpha}\right)+\sum_{\substack{m, k, l \\
m+k+l=\alpha}} P_{m, k, l} \\
& \cdot\left[\left(2+(a-2) 2^{2 m+k}\right)\left(2(3)^{k}+(b-2) 2^{2 k}\right) 2^{3 m+2 k}+\left(2+(a-2) 2^{m}\right)\left(2+(b-2) 2^{2 k+l}\right)\right] \text {, } \\
& U B_{T}=\sum_{\substack{m, k, l \\
m+k+l=\alpha}} P_{m, k, l}\left[2^{2 l}\left(2+(a-2) 2^{2 m+k}\right)\left(2(3)^{k}+(b-2) 2^{2 k}\right)+2^{3 m+2 k}\left(2+(a-2) 2^{m}\right)\right. \\
& \left.+2^{3 m+3 k}\left(2+(a-2) 2^{m}\right)\left(2+(b-2) 2^{2 k+l}\right)\right] \\
& +\left[\left(\sum_{i=0}^{\alpha}\left(\begin{array}{c}
\alpha \\
i
\end{array}\right) 2^{\alpha-i+1}\left(2+(b-2) 2^{i}\right)\right)+2^{3 m+2 k}\left(2+(a-2) 2^{m}\right)+b 2^{2 \alpha}\right]\left(2(3)^{\alpha}+(a-2) 2^{\alpha}\right) .
\end{aligned}
$$

\section{Conclusion}

For researchers, determining the bounds for pertinent topological index is always intriguing and attractive problem. In [45], Liu et al. proposed an open problem regarding GRI of four operations ( $F$-sum) on graphs. This paper, potentially, addressed and figured out the bounds on GRI for the
F-sum graphs $\Gamma_{1}{ }_{S} \Gamma_{2}, \Gamma_{1}+{ }_{R} \Gamma_{2}, \Gamma_{1}+{ }_{Q} \Gamma_{2}$, and $\Gamma_{1}+{ }_{T} \Gamma_{2}(\alpha \in \mathbb{N})$ in terms of eminent TIs of their base graphs and graph parameters. Several examples for different combinations of base graphs $\Gamma_{1}$ and $\Gamma_{2}$ along with different parameter $\alpha$ are explored. It can be observed that bounds obtained performed well when compared with exact value. It is worth mentioning that with the help of these four graph 
operations, one can construct the molecular graph of his own choice and GRI quantifies information of resulting molecular graph. Computing the bounds of GA-index and ABC-index (variations of GRI) for the F-sum graphs could be an interesting problem for future investigation.

\section{Abbreviation}

TI:

QSPR: Quantitative structure property relationships

QSAR: Quantitative structure activity relationships

GA-Index: Geometric arithmetic index

FGZI: $\quad$ First general Zagreb index

ABC-Index: Atom bond connectivity index

GRI: General Randić index

SCI: $\quad$ Sum-connectivity index

GSCI: General sum-connectivity index.

\section{Data Availability}

All data are included within this paper. However, the reader may contact the corresponding author for more details of the data.

\section{Conflicts of Interest}

The authors declare that they have no conflicts of interest.

\section{Acknowledgments}

This research was supported by the funding of Hefei University Annual Academy Research Development Fund Project (Natural Science) (no. 18ZR12ZDA).

\section{References}

[1] R. Gozalbes, J. Doucet, and F. Derouin, “Application of topological descriptors in QSAR and drug design: history and new trends," Current Drug Target-Infectious Disorders, vol. 2, no. 1, pp. 93-102, 2002.

[2] H. González-Díaz, S. Vilar, L. Santana, and E. Uriarte, "Medicinal chemistry and bioinformatics-current trends in drugs discovery with networks topological indices," Current Topics in Medicinal Chemistry, vol. 7, pp. 1025-1039, 2007.

[3] A. R. Matamala and E. Estrada, "Generalised topological indices: optimisation methodology and physico-chemical interpretation," Chemical Physics Letters, vol. 410, no. 4-6, pp. 343-347, 2005.

[4] G. Rücker and C. Rücker, "On topological indices, boiling points, and cycloalkanes," Journal of Chemical Information and Computer Sciences, vol. 39, no. 5, pp. 788-802, 1999.

[5] F. Yan, Q. Shang, S. Xia, Q. Wang, and P. Ma, "Application of topological index in predicting ionic liquids densities by the quantitative structure property relationship method," Journal of Chemical \& Engineering Data, vol. 60, no. 3, pp. 734-739, 2015.

[6] L. H. Hall and L. B. Kier, Molecular Connectivity in Chemistry and Drug Research, Academic Press, Boston, MA, USA, 1976.

[7] M. V. Diudea, QSPR/QSAR Studies by Molecular Descriptors, NOVA, New York, NY, USA, 2001.

[8] J. Devillers and A. T. Balaban, Topological Indices and Related Descriptors in QSAR and QSPR, Gordon \& Breach, New York, NY, USA, 1999.
[9] R. Todeschini, V. Consonni, R. Mannhold, H. Kubinyi, and H. Timmerman, Handbook of Molecular Descriptors, Wiley VCH, Weinheim, Germany, 2002.

[10] I. Gutman and O. Polansky, Mathematical Concepts in Organic Chemistry, Springer-Verlag, Berlin, Germany, 1986.

[11] I. Gutman and N. Trinajstić, "Graph theory and molecular orbitals. Total $\varphi$-electron energy of alternant hydrocarbons," Chemical Physics Letters, vol. 17, no. 4, pp. 535-538, 1972.

[12] X. Li and J. Zheng, "A unified approach to the extremal trees for different indices," MATCH Communications in Mathematical and in Computer Chemistry, vol. 54, pp. 195-208, 2005.

[13] B. Furtula and I. Gutman, "A forgotten topological index," Journal of Mathematical Chemistry, vol. 53, no. 4, pp. 11841190, 2015.

[14] B. Bollobás and P. Erdös, "Graphs of extremal weights," Ars Combinatoria, vol. 50, pp. 225-233, 1998.

[15] M. Randic, "On characterization of molecular branching," Journal of the American Chemical Society, vol. 97, pp. 6609$6615,1975$.

[16] B. Zhou and N. Trinajstić, "On general sum-connectivity index," Journal of Mathematical Chemistry, vol. 47, no. 1, pp. 210-218, 2010.

[17] B. Zhou and N. Trinajstić, "On a novel connectivity index," Journal of Mathematical Chemistry, vol. 46, no. 4, pp. 12521270, 2009.

[18] B. Lučić, N. Trinajstić, and B. Zhou, "Comparison between the sum-connectivity index and product-connectivity index for benzenoid hydrocarbons," Journal of Mathematical Chemistry, vol. 475, pp. 146-148, 2009.

[19] I. Gutman, "Degree-based topological indices," Croatica Chemica Acta, vol. 86, no. 4, pp. 351-361, 2013.

[20] S. Akhter and M. Imran, "On molecular topological properties of benzenoid structures," Canadian Journal of Chemistry, vol. 94, no. 8, pp. 687-698, 2016.

[21] K. C. Das and I. Gutman, "Some properties of the second zagreb index," Journal of Mathematical Chemistry, vol. 52, pp. 103-112, 2004.

[22] M. H. Khalifeh, H. Yousefi-Azari, and A. R. Ashrafi, "The first and second zagreb indices of some graph operations," Discrete Applied Mathematics, vol. 157, no. 4, pp. 804-811, 2009.

[23] M. Liu and B. Liu, "Some properties of the first general zagreb index," Journal of Mathematical Chemistry, vol. 47, pp. 285294, 2010.

[24] J. M. Xu, Topological Structure and Analysis of Interconnection Networks, Kluwer Academic, Dordrecht, MA, USA, 2001.

[25] M. Eliasi and B. Taeri, "Four new sums of graphs and their Wiener indices," Discrete Applied Mathematics, vol. 157, no. 4, pp. 794-803, 2009.

[26] Z. Yarahmadi, S. Moradi, and T. Došlić, "Eccentric connectivity index of graphs with subdivided edges," Electronic Notes in Discrete Mathematics, vol. 45, pp. 167-176, 2014.

[27] D. M. Cvetkocić, M. Doob, and H. Sachs, Spectra of Graphs: Theory and Application, Academic Press, New York, NY, USA, 1980.

[28] J.-B. Liu, J. Zhao, and Z.-Q. Cai, "On the generalized adjacency, Laplacian and signless Laplacian spectra of the weighted edge corona networks," Physica A: Statistical Mechanics and Its Applications, vol. 540, p. 123073, 2020.

[29] J.-B. Liu, C. Wang, S. Wang, and B. Wei, "Zagreb indices and multiplicative zagreb indices of eulerian graphs," Bulletin of the Malaysian Mathematical Sciences Society, vol. 42, no. 1, pp. 67-78, 2019.

[30] J.-B. Liu, J. Zhao, H. He, and Z. Shao, "Valency-based topological descriptors and structural property of the 
generalized sierpiński networks," Journal of Statistical Physics, vol. 177, no. 6, pp. 1131-1147, 2019.

[31] B. Furtula and I. Gutman, "Comparing energy and Randic energy," Macedonian Journal of Chemistry and Chemical Engineering, vol. 32, no. 1, pp. 117-123, 2013.

[32] I. Gutman, B. Furtula, and V. Katanić, "Randić index and information," AKCE International Journal of Graphs and Combinatorics, vol. 15, no. 3, pp. 307-312, 2018.

[33] Y. Ma, S. Cao, Y. Shi, I. Gutman, M. Dehmer, and B. Furtula, "From the connectivity index to various Randić-type descriptors," MATCH Communications in Mathematical and in Computer Chemistry, vol. 80, pp. 85-106, 2018.

[34] X. Li and Y. Shi, "A survey on the Randić index," MATCH Communications in Mathematical and in Computer Chemistry, vol. 59, no. 1, pp. 127-156, 2008.

[35] W. Yan, B.-Y. Yang, and Y.-N. Yeh, "The behavior of Wiener indices and polynomials of graphs under five graph decorations," Applied Mathematics Letters, vol. 20, no. 3, pp. 290-295, 2007.

[36] H. Wiener, "Structural determination of paraffin boiling points," Journal of the American Chemical Society, vol. 69, no. 1, pp. 17-20, 1947.

[37] X. Li, X. Wang, and B. Wei, "On the lower and upper bounds for general randić index of chemical ( $n$-m) graphs," MATCH Communications in Mathematical and in Computer Chemistry, vol. 52, pp. 157-166, 2004.

[38] L. Shi, "Bounds on Randić indices," Discrete Mathematics, vol. 309, no. 16, pp. 5238-5241, 2009.

[39] X.-F. Pan, H. Liu, and M. Liu, "Sharp bounds on the zerothorder general Randić index of unicyclic graphs with given diameter," Applied Mathematics Letters, vol. 24, no. 5, pp. 687-691, 2011.

[40] A. Ali, I. Gutman, E. Milovanovic, and I. Milovanovic, "Sum of powers of the degrees of graphs: extremal results and bounds," MATCH Communications in Mathematical and in Computer Chemistry, vol. 80, pp. 5-84, 2018.

[41] S. Elumalai and T. Mansour, "On the general zeroth-order Randic index of bargraphs," MATCH Communications in Mathematical and in Computer Chemistry, vol. 2, pp. 6-9, 2019.

[42] M. K. Jamil, I. Tomescu, M. Imran, and A. Javed, "Some bounds on zeroth-order general Randic index," Mathematics, vol. 8, 2020.

[43] H. Deng, D. Sarala, S. K. Ayyaswamy, and S. Balachandran, "The Zagreb indices of four operations on graphs," Applied Mathematics and Computation, vol. 275, pp. 422-431, 2016.

[44] M. Imran, S. Baby, H. M. A. Siddiqui, and M. K. Shafiq, "On the bounds of degree-based topological indices of the cartesian product of F-sum of connected graphs," Journal of Inequalities, vol. 305, pp. 1-14, 2017.

[45] J.-B. Liu, S. Javed, M. Javaid, and K. Shabbir, "Computing first general Zagreb index of operations on graphs," IEEE Access, vol. 7, pp. 47494-47502, 2019.

[46] M. Ahmad, M. Saeed, M. Javaid, and M. Hussain, "Exact formula and improved bounds for general sum-connectivity index of graph-operations," IEEE Access, vol. 7, pp. 167290167299, 2019. 